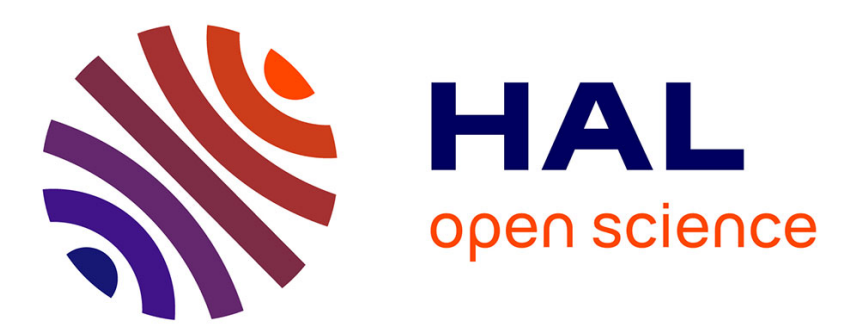

\title{
A review on applications of magnetoelectric composites: from Heterostructural uncooled magnetic sensors, Energy harvesters to Highly efficient power converters
}

\author{
Chung Ming Leung, Jiefang Li, D. Viehland, X. Zhuang
}

\section{- To cite this version:}

Chung Ming Leung, Jiefang Li, D. Viehland, X. Zhuang. A review on applications of magnetoelectric composites: from Heterostructural uncooled magnetic sensors, Energy harvesters to Highly efficient power converters. Journal of Physics D: Applied Physics, In press, 10.1088/1361-6463/aac60b . hal01801976

\author{
HAL Id: hal-01801976 \\ https://hal.science/hal-01801976
}

Submitted on 28 May 2018

HAL is a multi-disciplinary open access archive for the deposit and dissemination of scientific research documents, whether they are published or not. The documents may come from teaching and research institutions in France or abroad, or from public or private research centers.
L'archive ouverte pluridisciplinaire HAL, est destinée au dépôt et à la diffusion de documents scientifiques de niveau recherche, publiés ou non, émanant des établissements d'enseignement et de recherche français ou étrangers, des laboratoires publics ou privés. 


\title{
A review on applications of magnetoelectric composites: from Heterostructural uncooled magnetic sensors, Energy harvesters to Highly efficient power converters
}

\author{
Chung Ming Leung*, Jiefang Li, D. Viehland and X. Zhuang* \\ Department of Materials Science and Engineering, Virginia Tech, Blacksburg, Virginia \\ 24061, USA \\ *Corresponding author E-mail: cmleung@vt.edu; $\underline{\text { xin2@ @t.edu }}$
}

\begin{abstract}
Over the past two decades, magnetoelectric (ME) composites and their devices have been an important topic of research. Potential applications ranging from low power sensing to high power converters have been investigated. This review, first, begins with a summary of multiferroic materials that work at room temperature. Such ME materials are usually in composites, and their ME effect generated as a product property of magnetostrictive and piezoelectric composite layers. After that, mechanisms, working principles, and applications of ME composites from heterostructural uncooled magnetic sensors, energy harvesters to highly efficient power converters will be discussed. First, the development of ME sensors in terms of materials and structures to enhance their sensitivities and to reduce noise level is reviewed and discussed. Second, the structure of ME-based energy harvesters is discussed and summarized. Third, the development ME gyrators is summarized for power applications, including the current/voltage conversion, power efficiency, power density and figures of merit. Results demonstrate that our ME gyrator has the ability to satisfy the needs of power conversion with superior efficiency ( $>90 \%$ ), offering potential uses in power electronic applications.
\end{abstract}




\section{Introduction}

The term "multiferroic" defines a coexistence of magnetic and electric orderings in a solid. These orderings can polarize as either linear or nonlinear functions in response to an external field: including magnetization $(M)$ and its conjugate magnetic field $(H)$, and polarization $(P)$ and its conjugate electric field $(E)$. The cross-relations between magnetic properties $(M(H))$ and electric properties $(P(E))$ define the magnetoelectric (ME) effect. This cross-coupling results in a change in $P$ due to an applied $H$ (direct ME effect) or a change in $M$ due to an applied $E$ (converse effect). In this case, both the magnetic and electric properties can be mutually controlled. The concept of a ME effect was first suggested by Röntgen in 1888 [1]. He discovered that a dielectric material could be magnetized when placed in a magnetic field. Six years later, Curie suggested that some crystals have intrinsic ME effects [2]. The term magnetoelectric (ME) was first used by Debye in 1926, after studying magnetic and electric field induced changes in dipolar paramagnetic molecules [3]. In 1960, Astrov reported the first observation of magnetic-field-induced electric polarization in antiferromagnetic single crystals of chromium oxide $\left(\mathrm{Cr}_{2} \mathrm{O}_{3}\right)$ [4,5]. The $\mathrm{ME}$ effect can be subcategorized by its partial differentials into direct and converse effects. The direct ME effect is represented by the ME field coefficient, $\alpha_{\mathrm{ME}}=\frac{d D}{d H}$. It is defined by a change in the dielectric displacement $(D)$ in response to an applied magnetic field $(H)$. The converse effect is represented by $\alpha_{C M E}=\frac{d M}{d V}$. It is defined by a change in the magnetization $(M)$ in response to an applied electric voltage $(V)$.

In the following sections of this article, we will discuss recent progress and present status in the development of bulk ME materials and their applications. We will summarize the results concerning ME composites of macro-laminates, together with background and history, and extend the discussions to recent progress. Detailed reviews of ME materials have previously been published [6-9]. Applications based on ME composites will be presented in more detail in subsequent sections of this review, along with examples of sensors, energy harvesters and power converters that have been recently investigated. 


\section{Background of ME Materials}

Single-phase ME materials have been widely studied in the past decades [10]. A critical limitation for their application is the low transition temperatures of either their ferroelectric or ferromagnetic order parameters, and a weak exchange between them: these prevent their useful applications. To overcome the limitations of single-phase materials, two-phase composites (ferromagnetic/ferroelectric) were first proposed by van Suchtelen in the 1970s [11]. Important advantages of two-phase materials promising practical applications of ME technology were an enhanced ME coefficient that was dramatically higher than single phase materials, as well as increased flexibilities in operational temperature ranges, materials design, manufacturability, and size. Subsequently, strong ME effects have been identified in bulk laminated composites.

In the direct ME effect, a magnetic field applied to the composite produces a mechanical stress/strain in the magnetostrictive phase. This stress/strain results in a mechanical input to the piezoelectric phase which induces a piezoelectric voltage/electric field output proportional to (and of the same frequency) as the input magnetic field. In the converse ME effect, an electric voltage/field applied to the composite results in a mechanical strain in the piezoelectric phase. This strain induces a mechanical stress in the magnetostrictive phase, resulting in a magnetization change. These ME effects can be generically expressed as:

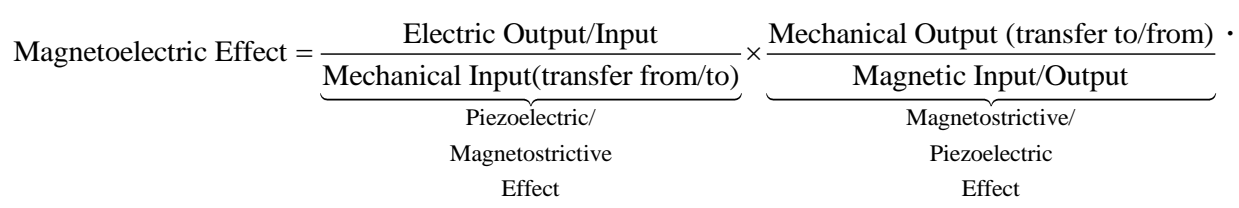

The intermediate strain/stress couplings between the two phases provide several beneficial features to ME composites, such as a stress enhancement of the ME effect at mechanical resonance, which is an oscillatory motion at a specific frequency. In this case, energy is stored as a mechanical form, but not dissipated by the oscillation. A mechanical quality factor $Q_{\text {mech }}$ defines the enhancement of the ME coupling at resonance. A standard figure of merit to evaluate the ME effect is the ME coefficient $\left(\alpha_{M \mathrm{E}}\right)$, which is defined as the value of the output electric voltage across a unit distance (i.e., field) in response to a unit AC magnetic field applied to the ME composite at a given frequency. Its units are $\mathrm{V} / \mathrm{cm} \cdot \mathrm{Oe}$. A larger value of $\alpha_{\mathrm{ME}}$ indicates stronger ME interactions between the two order parameters, i.e., $P$ to $H$ and/or $M$ to $E$.

In 1974, Boomgarrd et al. [12] fabricated a particulate ceramic composite of $\mathrm{BaTiO}_{3}$ $\mathrm{CoFe}_{2} \mathrm{O}_{4}$ based on a unidirectional solidification technique. A high value (at that time) of 
$\alpha_{\mathrm{E}}=0.13 \mathrm{~V} / \mathrm{cm}$ Oe was obtained at room temperature. This value of $\alpha_{\mathrm{E}}$ was $\sim 7 \times$ larger than that for single-phase $\mathrm{Cr}_{2} \mathrm{O}_{3}$ crystals $(0.02 \mathrm{~V} / \mathrm{cm} \mathrm{Oe})$. This is the first example of ME composites having a "product" tensor property, as originally proposed by van Suchtelen [11]. Many types of bulk two-phase ME composites based on product properties have since been fabricated and studied. ME composites can have many different phase interconnectivity structures between ferroelectric and ferromagnetic phases. Research has persisted in bulk composites for 40 years, however, there are several crucial reproducibility and reliability problems that remain unsolvable to date. These are: (1) difficulty in controlling the interconnectivity of the constituent phases; (2) chemical reactions between phases during the sintering process; (3) dielectric breakdown through the low-resistive magnetostrictive phase during poling of the piezoelectric phase; and (4) weak mechanical coupling between phases due to processinginduced mechanical defects such as pores and cracks, amongst other difficulties that have tended to reduce the ME effect and limit its practical applications.

To overcome these problems of bulk ME composites, laminated composites were first proposed by Ryu et al. in 2001 [13]. The laminate configuration was based on a sandwich structure. It combined a thickness-polarized $\mathrm{Pb}(\mathrm{Zr}, \mathrm{Ti}) \mathrm{O}_{3}(\mathrm{PZT})$ piezoelectric ceramic disk, with two radial magnetized Terfenol-D magnetostrictive alloys disks that were bonded by an epoxy adhesive. A large $\alpha_{M \mathrm{E}}=4.68 \mathrm{~V} / \mathrm{cm} \cdot \mathrm{Oe}$ was found at $f=1 \mathrm{kHz}$ at room temperature. A year later, the same investigators further enhanced the ME field coefficient to $10.3 \mathrm{~V} / \mathrm{cm} \cdot \mathrm{Oe}$ at an optimal magnetic bias field of $4 \mathrm{kOe}$ by changing the piezoelectric layer from a PZT ceramic to a $\mathrm{Pb}\left(\mathrm{Mg}_{1 / 3} \mathrm{Nb}_{2 / 3}\right) \mathrm{O}_{3}-\mathrm{PbTiO}_{3}$ (PMN-PT) piezoelectric crystal [13]. After these initial states, various other composites were reported using piezoelectric and magnetostrictive phases. In the ME laminate composite configuration, the ME coupling is strongly dependent on the magnetostrictive and piezoelectric phase layers, and the interfacial bonding between them. This is because mechanical stress/strain must be transferred between phases with high coupling and low losses. In particular, coupling is strongly dependent on the size and the working mode of both magnetostrictive and piezoelectric phases. The ME coefficient can be enhanced by changing the shape of the ME composite; accordingly, Dong et al proposed a sandwich structure in rectangular form and suggested that the best incident direction of a magnetic field was along its in-plane direction $[14,15]$.

Most ME laminated composites have been prepared based on a 2-2 (layer-layer) connectivity structure and bonded together by an epoxy adhesive or co-firing. Co-fire synthesis is a good technique to bond all-oxide magnetostrictive and piezoelectric layers. It has an 
advantage in cost-effectiveness due to labor-cost reductions. However, the high-temperature co-firing process imparts to the laminated composite several challenges, such as differential shrinkage rates and thermal expansion mismatches. As a consequence, epoxy bonding methods have become the preferred method for fabricated ME laminate composites. They also offer an additional advantage of a low bonding temperature. In the past ten years, the magnetostrictive materials, Terfenol-D ferrites and amorphous metals (Metglas), have been used in ME composites. The maximum effective piezomagnetic coefficient $\left(d_{\mathrm{eff}, \mathrm{m}}\right)$ of Metglas under an optimal magnetic bias is larger than that of Terfenol-D and ferrites. This enhanced effective piezomagnetic coefficient is due to the high permeability of Metglas. The magnetic susceptibility of certain Metglas foils is in order of $\sim 10000$, which results in an enhanced effective magneto-mechanical coupling. Due to high $d_{\mathrm{eff}, \mathrm{m}}$, and magnetic susceptibility coefficients, and low de-magnetization effects in Metglas, high ME coefficients have been achieved in laminate composites of their foils with piezoelectric layers. A colossal ME coefficient has been reported [16]. This extraordinary high ME coefficient in Metglas/PZT laminated composites has attracted the attention of the research community. It promises applications in magnetic sensors, energy harvesters and power converters [17].

Fe-rich Metglas are amorphous metal foils with a giant effective magnetostrictive coefficient. In 2006, Zhai et al. [16] reported a Metglas/polyvinylidene-fluoride (PVDF) based ME composite that enhanced the ME coefficient to $7.2 \mathrm{~V} / \mathrm{cm} \cdot \mathrm{Oe}$ at $1 \mathrm{~Hz}$, and up to $310 \mathrm{~V} / \mathrm{cm}$ at the electromechanical resonance (EMR) frequency under a relatively low dc magnetic bias field of 8 Oe. In the same year, a push-pull design in the piezoelectric layer of Metglas/PZT based ME laminated composites resulted in a further enhancement in the ME coefficient to 22 $\mathrm{V} / \mathrm{cm} \cdot \mathrm{Oe}$ (at $1 \mathrm{kHz}$ ) and $\sim 500 \mathrm{~V} / \mathrm{cm} \cdot \mathrm{Oe}$ (at resonant frequency) under a magnetic field bias of 5 Oe [17]. Later, in 2009, Dong [18] et al. showed longitudinal-longitudinal ( $L-L)$ $\mathrm{FeBSiC} / \mathrm{PZT} / \mathrm{FeBSiC}$ laminates that had a giant ME coefficient of $\sim 750 \mathrm{~V} / \mathrm{cm} \cdot \mathrm{Oe}$ at EMR due to a high mechanical quality factor in both the Metglas and hard-type PZT phases. More recently, by using Metglas and PMN-PT single crystals, the ME coefficient reached a value of $52 \mathrm{~V} / \mathrm{cm}$ Oe (at $1 \mathrm{kHz}$ ) [19] and $>1100 \mathrm{~V} / \mathrm{cm}$ Oe (at resonant frequency) [20] for push-pull mode ME laminated composites. The ME coefficient in the laminated composites can be further improved by different variables (i.e., size, poling optimization of the piezoelectric layer, annealing of Metglas, mechanical structure, etc.). In 2017, Lasheras et al. [21] analyzed the relationship between dimensions and ME coefficient. Three FeCoSiB/PVDF/FeCoSiB ME trilayer laminated composites were fabricated having lengths of 4, 3, $2 \mathrm{~cm}$. It was found that 
the ME coefficient was increased from $44 \mathrm{~V} / \mathrm{cm} \cdot \mathrm{Oe}(2 \mathrm{~cm})$ to $250 \mathrm{~V} / \mathrm{cm}$ Oe $(4 \mathrm{~cm})$ at the EMR. This variation of the ME coefficients with size was attributed to demagnetization effects. In addition to size contribution, Wang et al. [22] showed a 1.4× enhancement in the ME coefficient from $31 \mathrm{~V} / \mathrm{cm} \cdot$ Oe to $42 \mathrm{~V} / \mathrm{cm} \cdot O e$ by optimization of the poling temperature and $E$-field ramp rate for PMN-PT. A significant enhancement of the longitudinal piezoelectric $d_{33}$ and $k_{33}$ coefficients was reported when the poling temperature was increased to $120{ }^{\circ} \mathrm{C}$. In 2013 , Kirchhof, et al. [23] reported a high bending mode ME coefficient of $20000 \mathrm{~V} / \mathrm{cm}$ Oe (EMR) in $\mathrm{FeCoSiB} / \mathrm{AlN}$ composites that was measured in a vacuum. Chu et al. [24] recently reported longitudinal-transverse $(L-T)$ mode ME composites having a 1-D connectivity that consists of PMN-PT and laser-treated amorphous FeBSi Metglas ribbons, which had an ME coefficient of $7000 \mathrm{~V} / \mathrm{cm} \cdot \mathrm{Oe}(\mathrm{EMR})$. This large resonance ME coefficient is attributed to the enhancement of the mechanical quality factor $\left(Q_{\text {mech }}\right)$ of Metglas layers after laser annealing, which is hardened due to crystallization occurring in the Metglas. Thus, the total $Q_{\text {mech }}$ of ME composites was enhanced from 200 to 320 .

Ferrites are also good magnetostrictive candidate materials in both bulk and laminated ME composites. In 2003, Bichurin et al. [25] reported $\mathrm{NiFe}_{2} \mathrm{O}_{4}$ (NFO)/PZT laminates that had ME coefficient of $23 \mathrm{~V} / \mathrm{cm}$ Oe (EMR), which was much higher than that previous reported for ferrite/PZT composites. In 2007, an enhanced ME coefficient of $45 \mathrm{~V} / \mathrm{cm}$ Oe (EMR) was reported in single-crystal-NZFO/PZT composites [26]. In addition, in 2009, Rananaa et al. [27] proposed a bulk composite with a high ME coefficient of $3.15 \mathrm{~V} / \mathrm{Oe} \mathrm{cm}$ (non-resonance) that consisted of $\mathrm{Ni}_{0.93} \mathrm{Co}_{0.15} \mathrm{Cu}_{0.02} \mathrm{Fe}_{1.9} \mathrm{O}_{4}$ (NCCFO) and PZT layers. Later, in 2017, Leung et al. showed $\mathrm{Ni}_{0.8} \mathrm{Zn}_{0.3} \mathrm{Fe}_{2} \mathrm{O}_{4}$ (NZFO) ferrite/PZT/NZFO ferrite laminates that had a much larger ME coefficient of $632 \mathrm{~V} / \mathrm{cm} \cdot \mathrm{Oe}(\mathrm{EMR})$ due to the high mechanical quality factor in both the NZFO/PZT layers [28]. Figure 1 summarizes a list of several ME coefficients that have been reported in the last decade for different configurations and materials, working under nonresonance and resonance conditions. 
Fig.1. Reported values of non-resonance and resonance ME electric field coefficients for various types of materials $[24,23,28-32,19,33,18,27,25]$, respectively.

Processing and geometry variables have been studied for ME property optimization. For FeSiB Metglas layers, Freeman et al. [34] found that annealing in a vacuum environment at 400 ${ }^{\circ} \mathrm{C}$ for 30 min under a magnetic field of $160 \mathrm{mT}$ resulted in an optimal ME coefficient of $6.1 \mathrm{~V} / \mathrm{cm} \cdot \mathrm{Oe}$ in Metglas/PZT composites. Other studies focused on the structural design of ME laminated composites. Enhancements of the ME coefficient have also been found using planeshaped laminates that combine $L-T$ and shear-shear modes. Xin et al. [35] demonstrated a $2 \times$ enhancement of the ME coefficient in FeBSiC/PZT laminates, where both ends of the Metglas foils were mechanically clamped by glass to achieve a shear mode configuration.

Semi-ring ME composite structures have been constructed [36] of Ni/PZT/Terfenol-D, as shown in Fig. 2. Since Ni is a negative magnetostrictive material and Terfenol-D is a positive one, the new semi-ring structure exhibited a 1.2× higher ME coefficient ( 36.1 to $40.5 \mathrm{~V} / \mathrm{cm}$ Oe) than one of the same configuration that used only positive magnetostrictive materials. Also, a type of ME structure was designed using Terfenol-D, PZT and a nonmagnetic-dielectric trestle which was configured in an A-line type with a shape knuckle joint assembly [37]. This micotrestle had a maximum ME coefficient $\sim 2 \times$ higher than that for conventional laminated ones. 
(a)
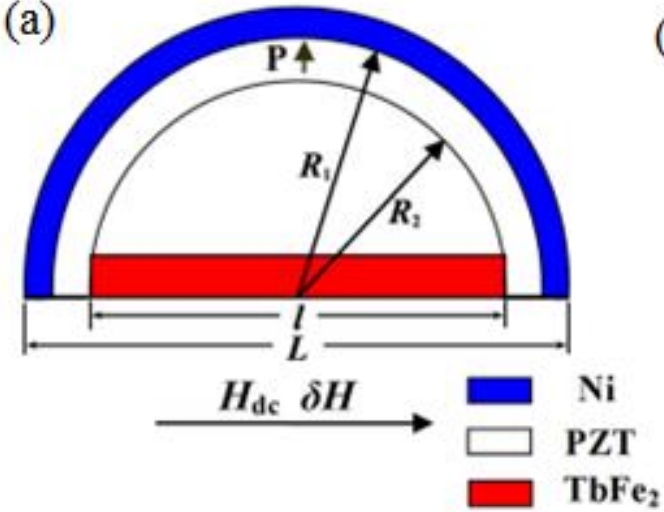

(b)

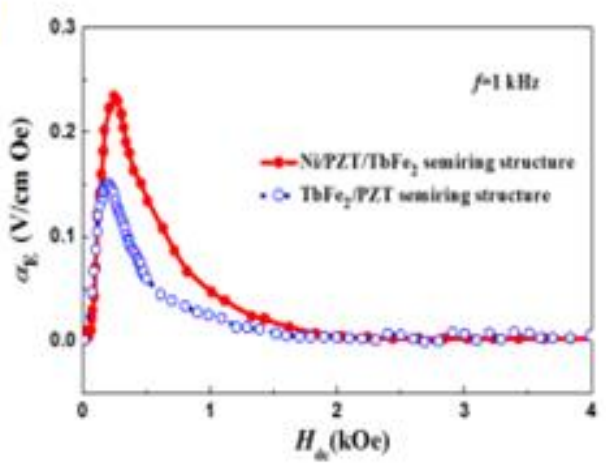

Fig. 2. (a) Schematic geometry arrangement of the Ni/PZT/TbFe2 semiring structure. (b ) Bias magnetic field $\boldsymbol{H}_{\mathrm{dc}}$ dependence of ME voltage coefficient $\boldsymbol{\alpha}_{\mathrm{E}}$ at $\boldsymbol{f}=\mathbf{1} \mathrm{kHz}$ [36]. Reproduced from Journal of Applied Physics, 119, 034102 (2016), with the permission of AIP Publishing.

Using interdigital electrodes, $L-L$ multiple-push pull configurations have been introduced into ME composites. Magnetostrictive FeBSiC alloy ribbons laminated with piezoelectric PZT fibers exhibited a strong resonant ME voltage coefficient of $750 \mathrm{~V} / \mathrm{cm}-\mathrm{Oe}$ (EMR), as shown in Figs. 3 and 4 [18]. The strong enhancement of the ME coefficient can be attributed to the high mechanical quality factor $Q_{\mathrm{m}}$ of the Metglas ribbon and hard-type PZT ceramic fiber.

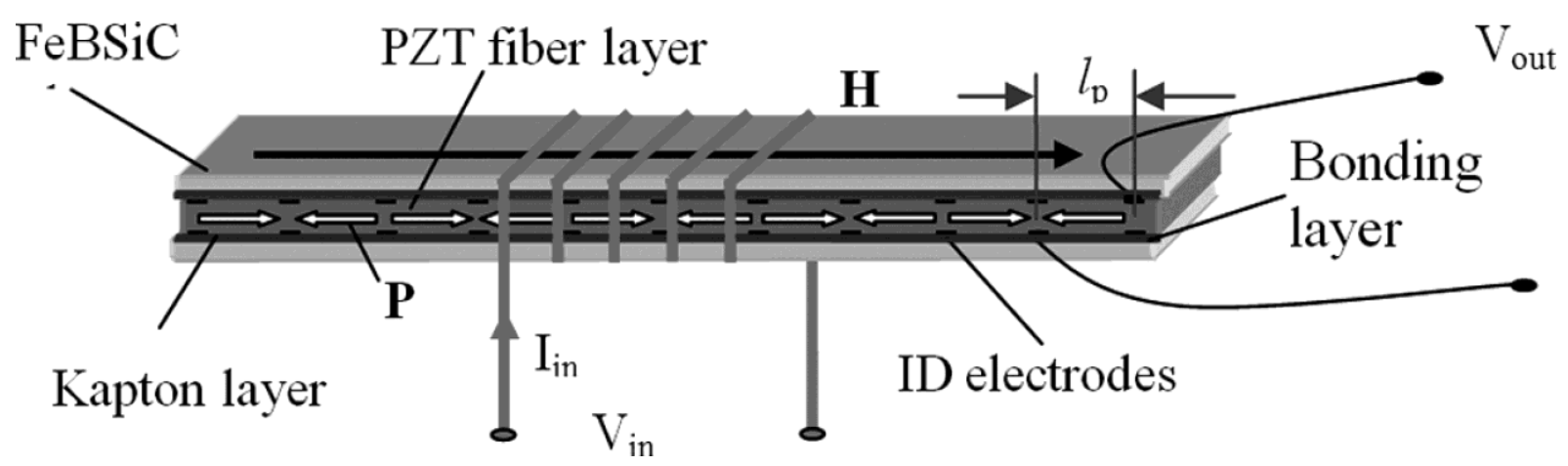

Fig. 3. Magnetoelectric transformer with a 5-layer laminate configuration (2-layer Metglas/1-layer PZTfiber/2-layer Metglas). The size of the laminate as $100 \mathrm{~mm}$ in length, $12 \mathrm{~mm}$ in width, and $0.36 \mathrm{~mm}$ in thickness [18]. Reproduced from IEEE Transactions on Ultrasonics, Ferroelectrics, and Frequency Control, 56, 1124-7 (2009), with the permission of IEEE. 


\section{Applications of ME composites}

Fig. 4. Magnetoelectric voltage coefficient as a function of magnetic field frequency at $H_{\mathrm{dc}}=5 \mathrm{Oe}$ [18]. Reproduced from IEEE Transactions on Ultrasonics, Ferroelectrics, and Frequency Control, 56, 1124-7 (2009), with the permission of IEEE.

There are several characteristic properties of ME composites that are potentially enabling for applications. The first is a characteristic curve for the ME coefficient as a function of magnetic bias [15]. High magnetically induced strains exist in some soft magnetostrictive materials. A magnetic bias is required to align the magnetization vector along the easy direction to maximize the induced strain. Second, the ME coefficient at the mechanical resonant frequencies can be order(s) of magnitude higher than that at sub-resonant ones. Third, the power loss (or dissipation) in ME composites influences the ME coupling, having an obvious significant effect on the efficiency. Last, ME composites have a tunability feature that results from the shift of the resonant frequency, impedance and output voltage characteristics with bias. These four characteristic features enable potential applications of ME composites in memory devices, gyrators, magnetic sensors, filters, and resonators.

Memory devices (Fig. 5) with magnetic/electric write and read have been an important developing focus of ME materials that has attracted the attention of investigators [38]. A memory device based on ME composites could enable low cost and effective write/read speeds at low power consumption. However, a full $180^{\circ}$ switching of the magnetization may be 
difficult to achieve by strain mediated coupling [39]. The ability of cross-controlling magnetic and electric polarization may be a key for such switching for this application.

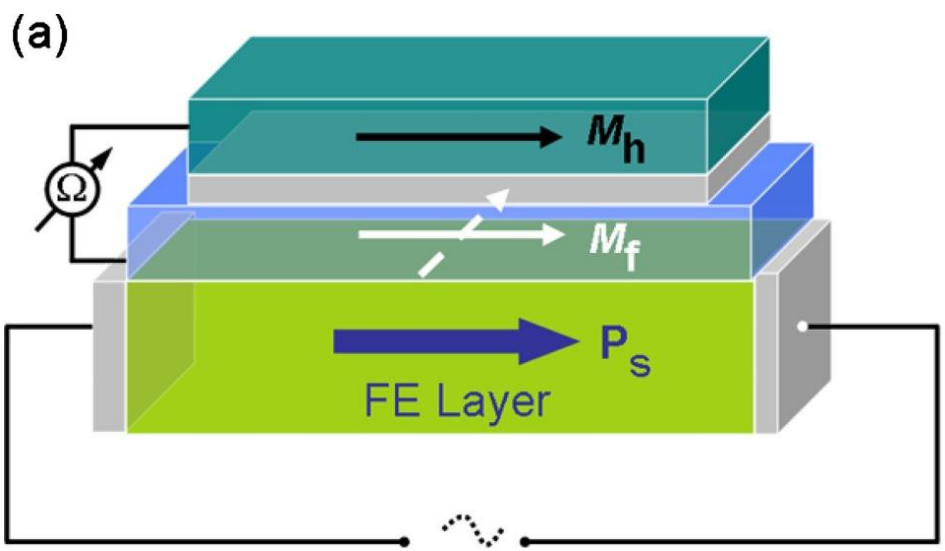

(b)

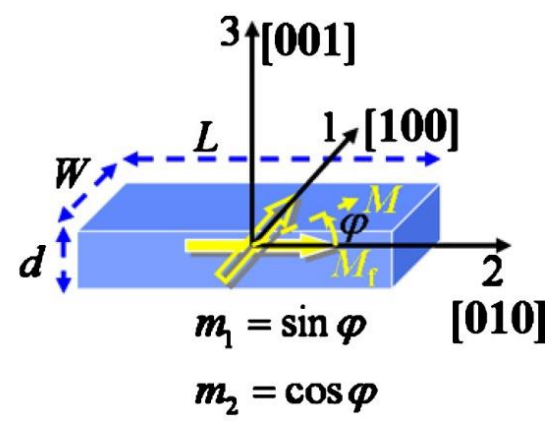

$\mathbf{E}$

Fig. 5. (a) Schematic diagram of a SME-RAM element. Mh and Mf show the initial magnetization orientations in the upper hard layer and the bottom free layer of a MTJ unit, respectively. A transverse electric field $\mathrm{E}$ is applied to the $\mathrm{FE}$ layer with a spontaneous polarization Ps to generate a $90^{\circ}$ in-plane magnetization switching. (b) the $90^{\circ}$ in-plane magnetization switching process in the free layer with its length $(L)$, width $(W)$, and thickness $(d)$ directions along the three principle crystal axes, i.e., the [010], [100], and [001], respectively [38]. Reproduced from Journal of Applied Physics, 107, 093912 (2010), with the permission of AIP Publishing.

ME composites with a coil carrying current have a gyration effect, which converts current into a voltage. The ME gyrator (Fig. 6) is a two-port four-wire device [40,41]. Calculations of the impedance parameters of this device have shown that an ideal gyrator element can be achieved when the square of the ME susceptibility is equal to the product of the permeability and permittivity in the ME composite (i.e. $\alpha_{m e}^{2}=\mu \varepsilon$ ) [41]. The current-to-voltage conversion, or trans-impedance, presents itself as a gyration coefficient. The ME coupling at the resonant frequency is a means to approach an ideal ME gyrator since power conversion is only appreciably enhanced at resonance: in this case, the equivalence of $\alpha_{m e}^{2}=\mu \varepsilon$ is approximately satisfied. A key feature of gyrators is the enhanced ME coupling at the mechanical resonant frequency. 


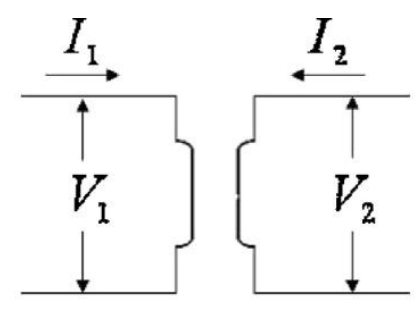

(a)

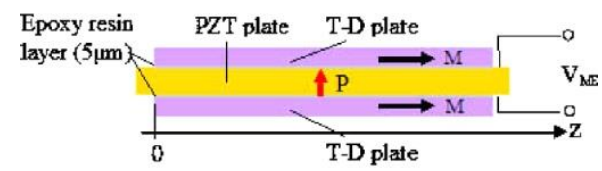

(b)

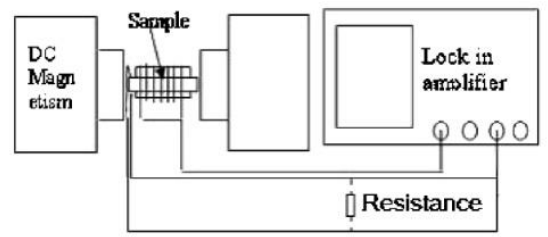

(c)

Fig. 6. (a) Gyrator equivalent circuit; (b) illustration of longitudinal-transverse or $L-T$ mode of a magnetoelectric laminate composite consisting of longitudinally poled $\mathrm{Pb}\left(\mathrm{Zr}_{\mathrm{x}}, \mathrm{Ti}_{1-\mathrm{x}}\right) \mathrm{O}_{3}$ layer sandwiched between two longitudinally magnetized Terfenol-D layers, epoxied together with a thin insulating resin layer; and (c) schematic illustrating the experiment setup [41]. Reproduced from Journal of Applied Physics, 100, 124509 (2006), with the permission of AIP Publishing.

Magnetic sensors based on ME composites offer a new generation of passive and active magnetic sensors [19,42-44], as summarized in Fig.7. Noise sources can be either extrinsic or intrinsic. The former is due to unwanted fluctuations that are incident on the ME sensor, i.e. magnetic and vibration fluctuations; however, the latter results from the internal power losses via a dissipative term (such as dielectric loss in piezo-phase). ME sensors have several characteristics which are the magnetic transfer function, the noise level and the equivalent magnetic noise (EMN) [45]. The transfer function is the sensing capacity, the noise level is the reliability of the detection and the EMN is the resolution of the sensors. The EMN is calculated by the noise spectral density over the transfer function. A lower level of EMN offers a better sensor sensitivity for measuring the strength of an unknown magnetic field. In addition to the ME coefficient that can enhance the transfer function, the fluctuation-dissipation term is also a key factor for ME sensors. 

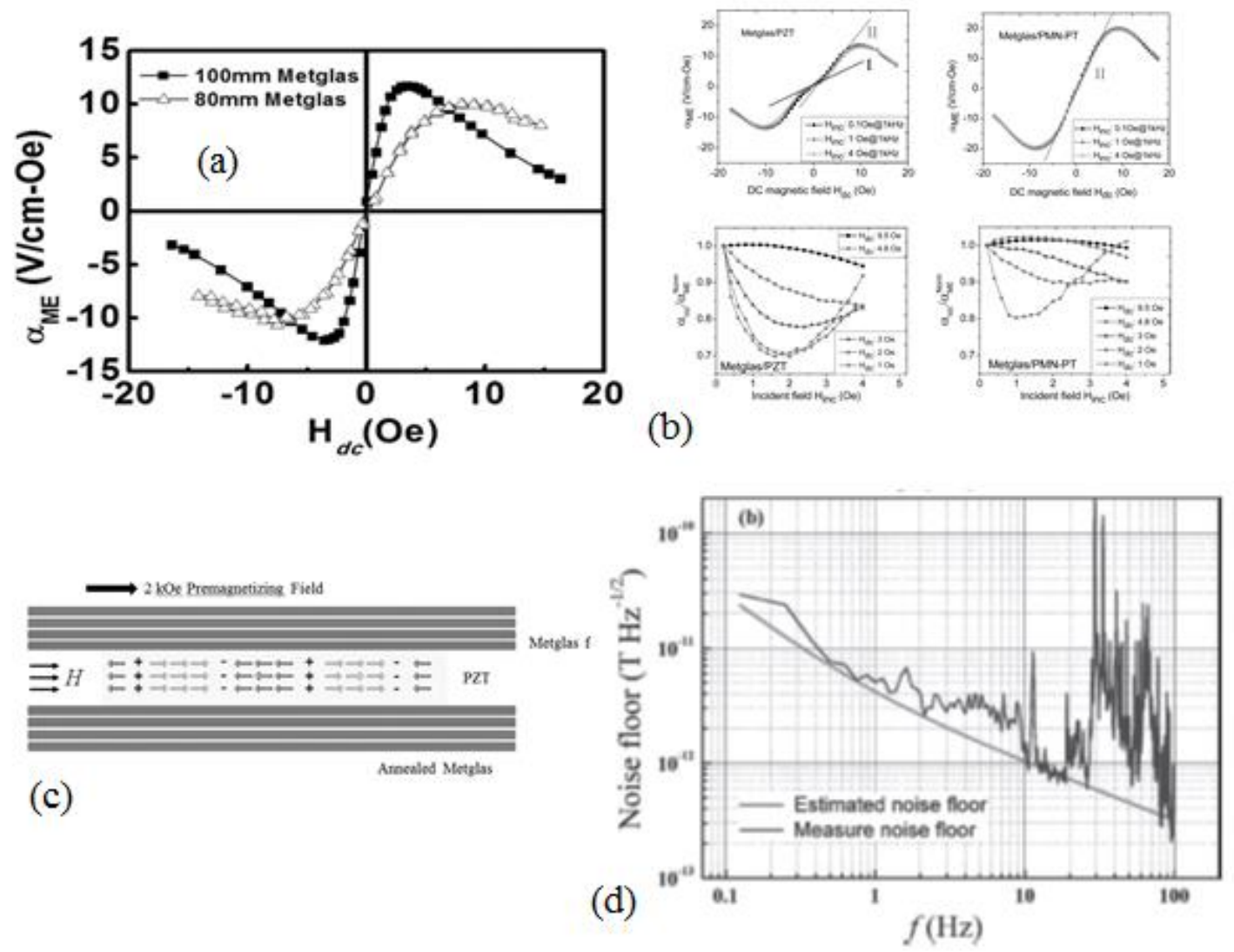

(c)
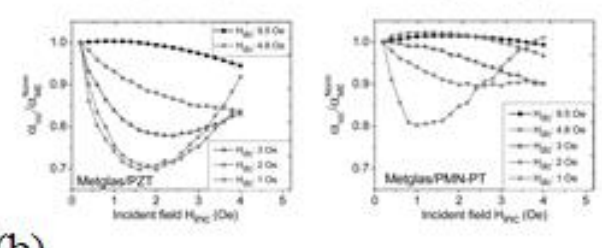

(b)

Fig. 7. Linear (a) and nonlinear (b) ME coefficients [42,43], (c) ME sensor in LL multiple push-pull configurations [44] and (d) equivalent magnetic noise spectral density [19]. Reproduced from Applied Physics Letters, 99, 153502 (2011), Journal of Applied Physics, 110114510 (2011), Applied Physics Letter, 102, 082404, and Advanced Materials, 23, 153502 (2011), with the permission of AIP Publishing and Wiley-VCH.

The narrow bandwidth of ME laminate resonators (Fig. 8-9) enables their application as ME tunable filters/resonators [46-48]. This is because the resonant frequency can be tuned by applying a magnetic or electric bias field. The signal/power is transmitted over an allowed bandwidth that is determined by the external field strength. Tunable filters/resonators have a certain relation with ME sensors, since the tunability of the resonant frequency can be expressed as a function of the magnetic field strength. Such ME filters/resonators are based on ferritepiezoelectric composites. Other features of the ferrites that allow the filter/resonator to work at ultrahigh frequencies are the tunability of the resonant frequency, impedance and $M E$ coefficient are key factors for tunable filters/resonators. 

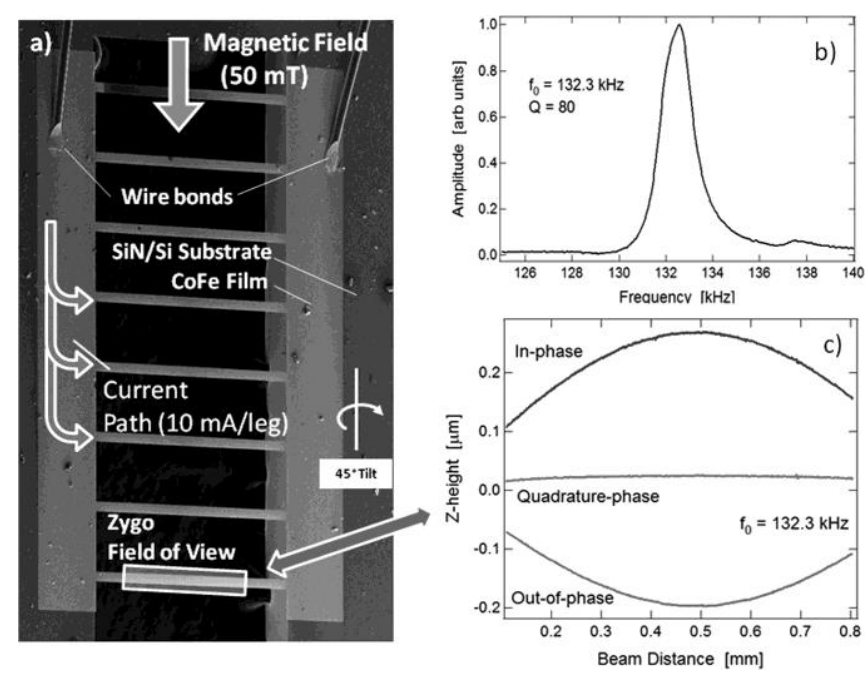

Fig. 8. SEM image of selected beam array showing $40 \mu \mathrm{m} \times 1 \mathrm{~mm}$ beam set at $45^{\circ}$ tilt; (b) Zygo beam resonance spectrum and (c) surface plot and lines can evaluation at named phase angles under Lorentz force excitation [46]. Reproduced from Applied Physics Letters, 104, 072408 (2014), with the permission of AIP Publishing.

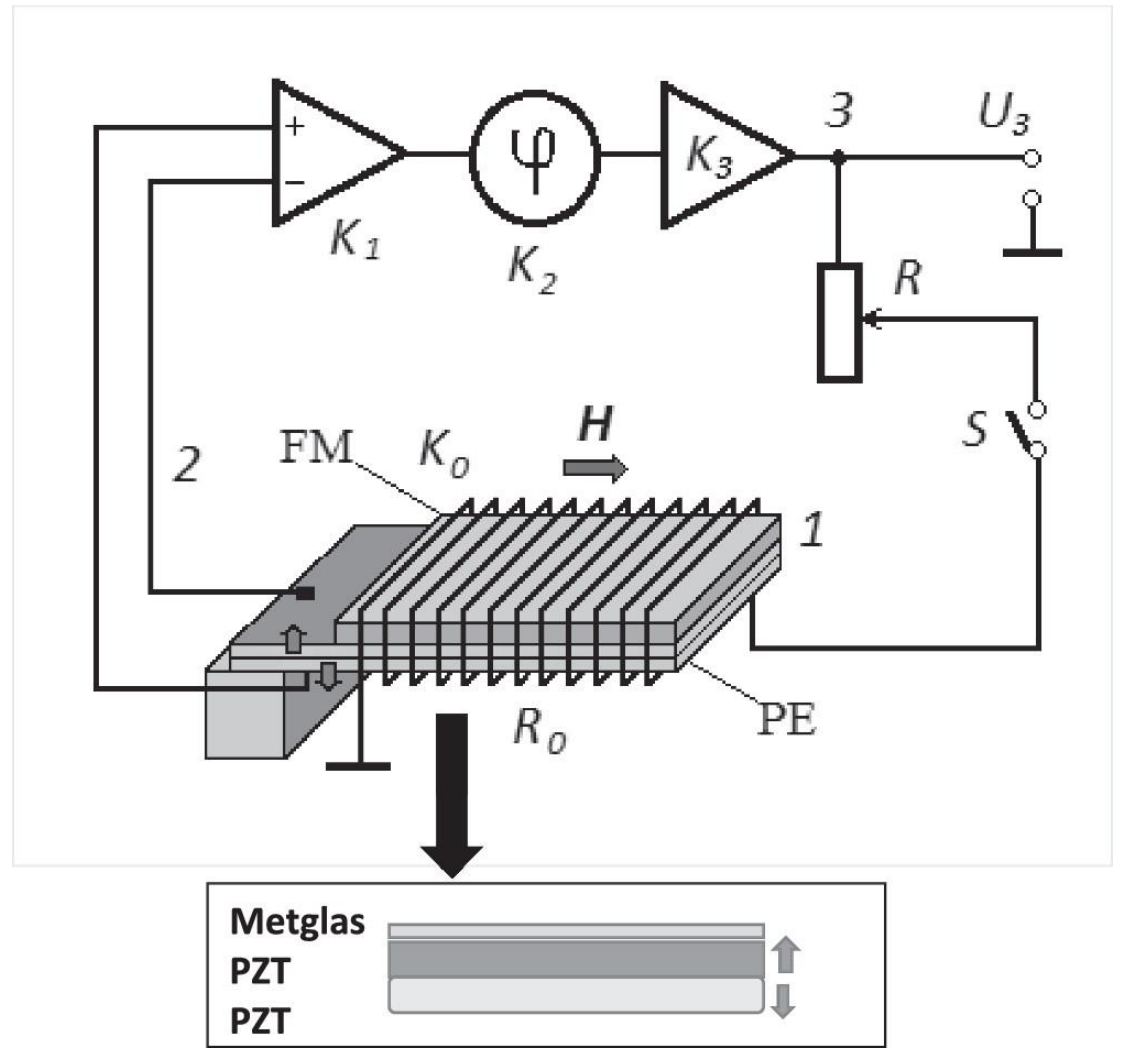

Fig. 9. Block-diagram of the signal generator with a magnetoelectric (ME) composite resonator (1) in the feedback loop (2). The composite is an epoxy bonded layered structure with a piezoelectric (PE) PZT bimorph and a ferromagnetic (FM) Metglas layer. Amplifiers and an RC-phase shifter are also shown. The inset shows the cross section of the composite with PZT bimorph and Metglas layer [48]. Reproduced from Applied Physics Letters, 108, 213502 (2016), with the permission of AIP Publishing. 
Both ME sensors and gyrators are electronics devices: the former transfers magnetic field/flux into electric signals, and the latter converts the current to a voltage based on transimpedance. The ME sensor can thus serve as a micro-power convertor at sub-resonant/resonant frequencies. When the magnetic power conversion is high, ME composite can detect a magnetic field with high sensitivity. ME gyrators can also be high-power converters working near their mechanical resonant frequency. When the power conversion is close to unity, a coil-ME structure can be considered as an ideal gyrator.

\section{$\underline{\text { Heterostructural uncooled magnetic sensors }}$}

Magnetic sensors that operate at room temperature with ultrahigh sensitivity have enabling applications for penetration imaging, and tracking and/or communications. Technical goals of ME sensors were to demonstrate a high sensitivity [49]. Target sensitivities from $1 \mathrm{fT} / \sqrt{ } \mathrm{Hz}$ to $1 \mathrm{p} \mathrm{T} / \sqrt{ } \mathrm{Hz}$ at $1 \mathrm{~Hz}$ have been sought. To approach this detection level, the noise source in ME sensors should be reduced, while amplifying the ME gain coefficients by an order of magnitude. Magnetic and piezoelectric materials with high conversion factors are desired by which to fabricate composites of high ME coefficients.

Our team has previously developed ME composites consisting of laminate layers to convert magnetic energy to electric energy, by using symmetric and asymmetric bending mode ME composites [50,52]. A 2-1 connectivity configuration consisting of 1-D piezo-fibers and 2D FeBSiC Metglas has been reported to have a high ME coefficient of $22 \mathrm{~V} / \mathrm{cm} \cdot \mathrm{Oe}$ (subresonance) and $1000 \mathrm{~V} / \mathrm{cm} \cdot \mathrm{Oe}(\mathrm{EMR})$. This enhancement was attributed to the colossal effective magnetostrictive coefficient in $\mathrm{FeBSiC}$ and the use of piezoelectric materials with high $d_{33, p}$ values in a longitudinal-longitudinal $(L-L)$ configuration [51], as shown in Fig. 3. This promised an unleashing of the potential of magnetoelectricity in laminate composites. In addition to the ME coefficient, the loss factor in the piezoelectric constituent phase is an important parameter by which to improve the detection capability. Magnetic sensors based on Metglas and PMN-PT single crystals laminates have shown an extremely low equivalent noise spectral density of $<10 \mathrm{pT} / \sqrt{ } \mathrm{Hz}$ at $1 \mathrm{~Hz}[19,54]$. With low power consumption and relatively small size, ME magnetic sensors have been considered as an alternative sensing technology with great potential to displace many existing magnetic sensors technologies. Enhancement of ME coefficients at the EMR pushes the noise floor of ME sensors below $1 \mathrm{pT} / \sqrt{\mathrm{Hz}}$ near the resonant frequency [53]. In addition, ME composites can harvest electromagnetic energy, when 
excited at its fundamental resonance. The associated amplifier can be charged by harvesting energy, which can enable self-powered ME sensors capable of long-term development [54].

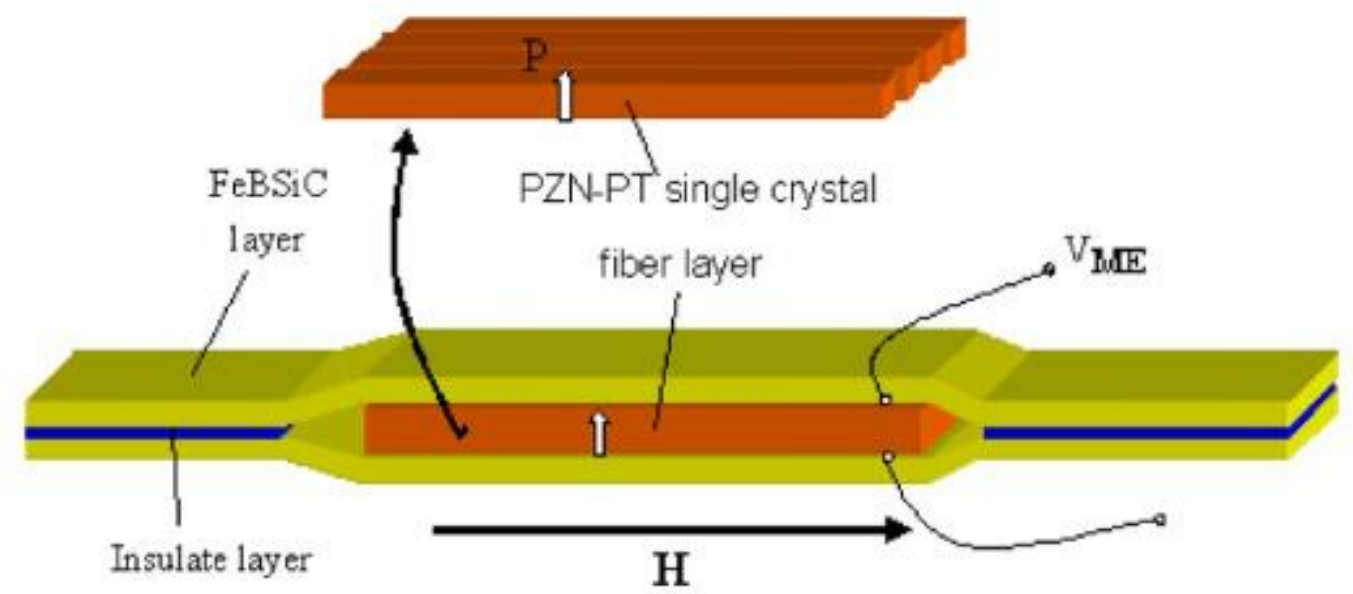

Fig. 10. Structure of FeBSiC/PZNPT-fiber laminate [51]. Reproduced from Applied Physics Letters, 91, 022915 (2007), with the permission of AIP Publishing.

The measurement of magnetic sensors with high sensitivity requires a measurement system with lower environmental noise sources. As such, the GREYC-CNRS research group has introduced a method for characterizing ME sensors at low frequency in a 6-layer magnetic shielded room, which has a noise level lower than SQUID sensitivity [45]. Using this measurement system, the noise contributions from several noise sources in both ME sensors and their conditioning detection circuits were studied and analyzed. The results identified that the intrinsic factor that may limit the ME sensor performance is the dielectric loss in the piezoelectric phase. This has been verified by both calculated and measured noise spectral densities. Frequency conversion techniques that shift the low-frequency signals to much higher ones near a carrier signal allows avoiding/reducing the dominant noise at low frequencies. The Army Research Lab (Adelphi, MD) has contributed to this technique by using flux concentrators that have single-support or double clamped ME films of Galfenol on PZT [55]. GRYEC realized a noise level of $30-40 \mathrm{pT} / \sqrt{ } \mathrm{Hz}$ using Metglas/piezo-fiber ME composites having an excitation carrier at the resonant frequency [56]. Self-biased and symmetric L-T mode ME sensors have been developed by Oakland University [57]. Self-biased sensors operate without magnetic bias, as shown in Fig. 11. The effect is due to a gradient magnetostriction between the two different magnetic phases: this provides sensitivities below $100 \mathrm{pT} / \sqrt{ } \mathrm{Hz}$ at 1 Hz. Measurements of sensors with different stacking configurations of piezoelectric PZT layers verified a volume scale effect for ME sensors [58]. One dimensional ME composites offer the possibility to overcome the magnetic demagnetization effect. Northeastern University 
developed a quasi-one dimension ME sensor consisting of a FeNi/PZT composite, which had a ME coefficient of $1.65 \mathrm{~V} / \mathrm{cm}-\mathrm{Oe}$ under zero magnetic bias [59].

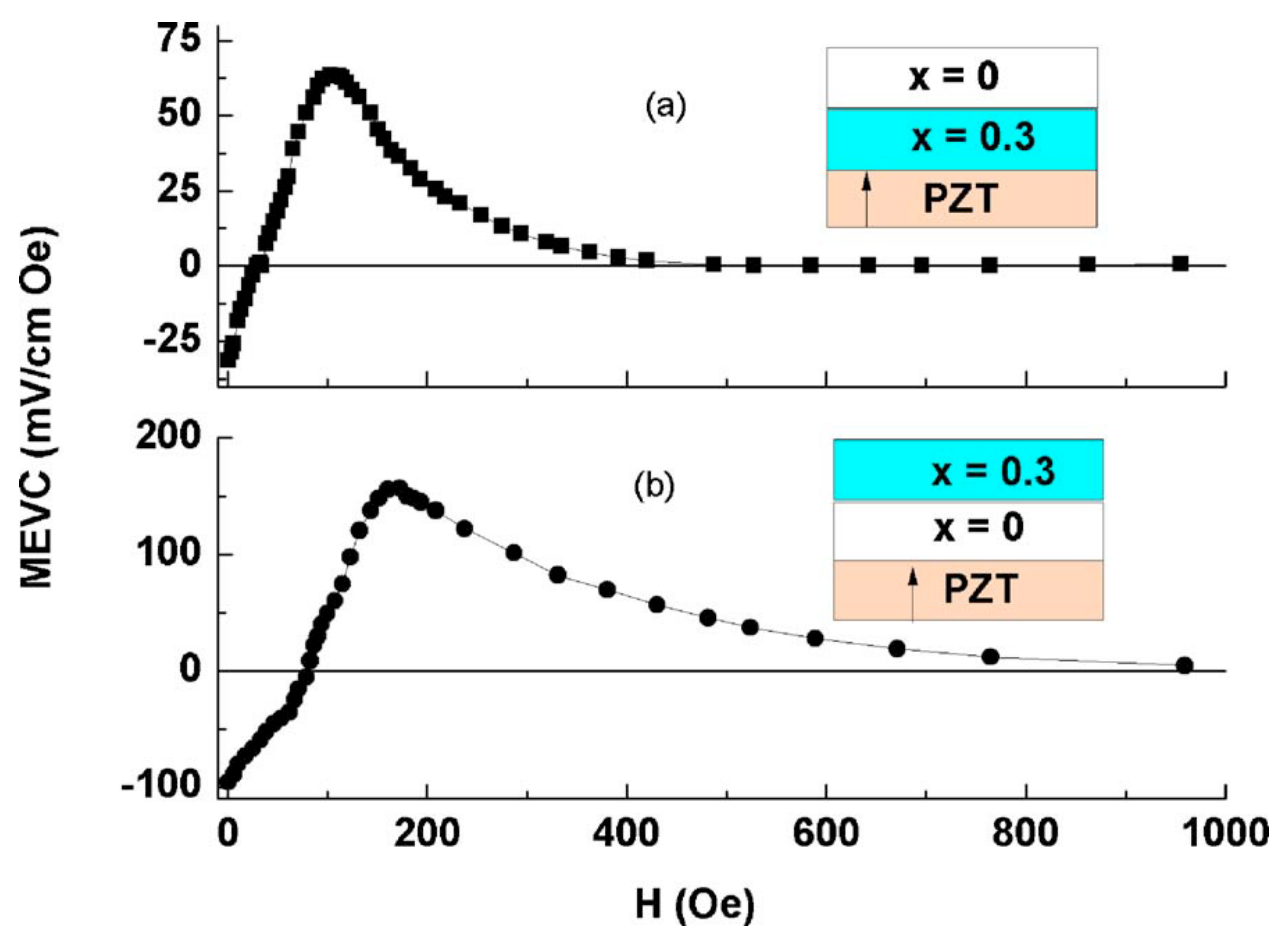

Fig. 11. Bias field dependence of MEVC in bilayers with equal volume of PZT and graded NZFO for (a) negative grading of $\boldsymbol{q}$ and (b) positive grading of $\boldsymbol{q}$ in the ferrite layer [57]. Reproduced from Applied Physics Letters, 96, 192502 (2010), with the permission of AIP Publishing.

Structure and geometry optimizations of ME composites have been investigated to enhance the ME sensitivity. This includes a volume scale effect and the optimal thickness ratio for various material combinations, in addition to the bonding in the intermediate layer(s). The ME effect was reported to be increased by means of an optimal mechanical coupling between the magnetostrictive and piezoelectric layers [60]. A specific thickness ratio between magnetic and piezoelectric phases was observed to maximize the ME coefficient. This ratio depended on the coefficients in the ME composite, such as the compliance module of both the magnetic and piezoelectric phases. Strong ME effects were reported for nickel-ferrite/PZT composites. The field sensing ability is related to the field coupling onto the magnetic phase. Thus, the magnetic field conversion for the volume effect needs to be considered for practical applications. The volume effect of the piezoelectric phase has been investigated as well. This includes stacked layers with several ME composites to increase the volume of the piezoelectric phase. An increased volume was identified to result in a lower equivalent magnetic noise level. This is due to the fact that the transfer function increases with increasing volume, but the noise increases as the root of the volume. An improved bonding condition results in a significant 
increase in the ME coefficient and a reduction in the equivalent magnetic noise floor. Optimization of the epoxy thickness increases the coupling between the magnetostrictive and piezoelectric layers $[61,62]$. Thus, a vacuum-bag curing technique was introduced in the ME sensor fabrication [61]. It has been reported that the ME coefficient is dependent on the thickness and Young's modulus of the bonding materials [63]. The ME coefficients decreased as the bonding layer thickness is increased, whereas Young's modulus was decreased.

Thin film ME resonators have been studied as magnetic field vector sensors. Sputtered Fe based alloys ( $\mathrm{TbFe}, \mathrm{FeCo}$ ) on PZT were investigated, which enhanced the magnetostrictive coefficient above that of Terfenol-D [64]. Interdigital electrodes have been deposited onto the structure of the ME thin films, which was reported to significantly improve the ME coefficient [65]. Recently, FeCoSiB/AlN thin film showed a ME coefficient as large as $6900 \mathrm{~V} / \mathrm{cm}$ Oe at the bending made mechanical resonant frequency, along with a limit of detection of 1 $\mathrm{pT} / \sqrt{\mathrm{Hz}}$ [66]. The volume effect of ME composite is an important parameter limiting the detection performance of magnetic sensors. The limit of detection of inverse bilayer magneto magnetoelectric thin films has been measured to reach $400 \mathrm{fT} / \sqrt{ } \mathrm{Hz}$ at their mechanical resonance, accompanied by a ME coefficient as high as $5000 \mathrm{~V} / \mathrm{cm}$ Oe [67]. When performed out-of-resonance, detection by thin film sensors falls behind the bulk ones. A suggested solution to enhance the detection ability is the realization of partially released symmetrical thin film composite sensors that would perform a longitudinal expansion of the sensor, rather than a bending mode.

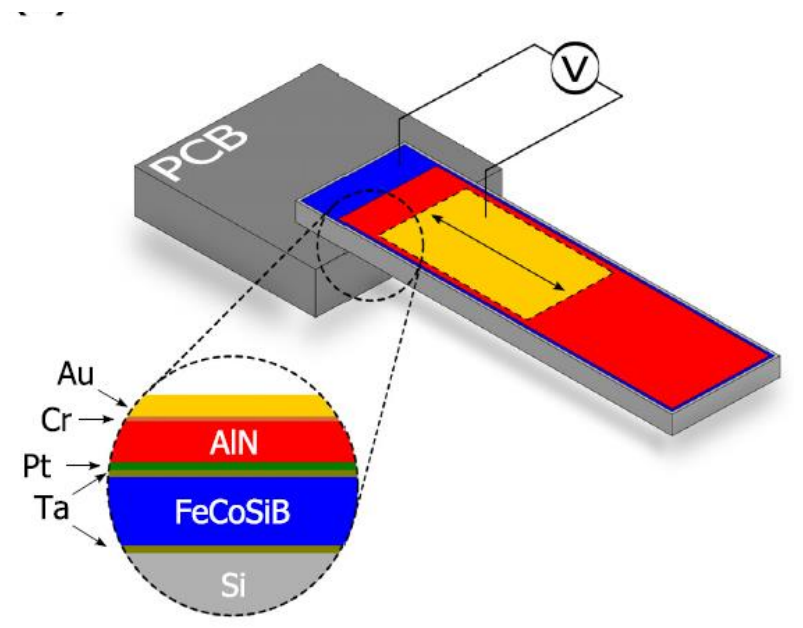

Fig. 12. Schematic illustration of thin film sensor [67]. Reproduced from Applied Physics Letters, 109, 022901 (2016), with the permission of AIP Publishing.

The piezoelectric phase in ME composites serves to convert a strain transmitted from the magnetostrictive phase into a voltage/charge output. Because the piezoelectric phase is also 
sensitive to vibration fluctuations, ME sensors have limitations in mechanically noisy environments [68]. Various vibration canceling methods have been studied to improve the sensing ability for real-world applications. These methods were based on built-in structures that separate magnetic and vibration induced signals generated by different modes, or by use of adaptive calculations to subtract the vibration noise using a reference vibration/acoustic sensor. Another approach to avoid the influence of vibrations is based on modulation/frequency conversion techniques $[69,70]$. A low-frequency signal modulates a carrier which is excited in the vicinity of the mechanical resonant frequency, where both the intrinsic noise and external fluctuations are weaker than the one at low frequencies. After a classical demodulation process, the low-frequency signals can be recovered from the sideband signals near the carrier. This working mode also enables quai-DC field detection capacities for ME composites [71-73]. Materials and structures that optimize the ME sensitivity based on nonlinearity have also been investigated [74-76].

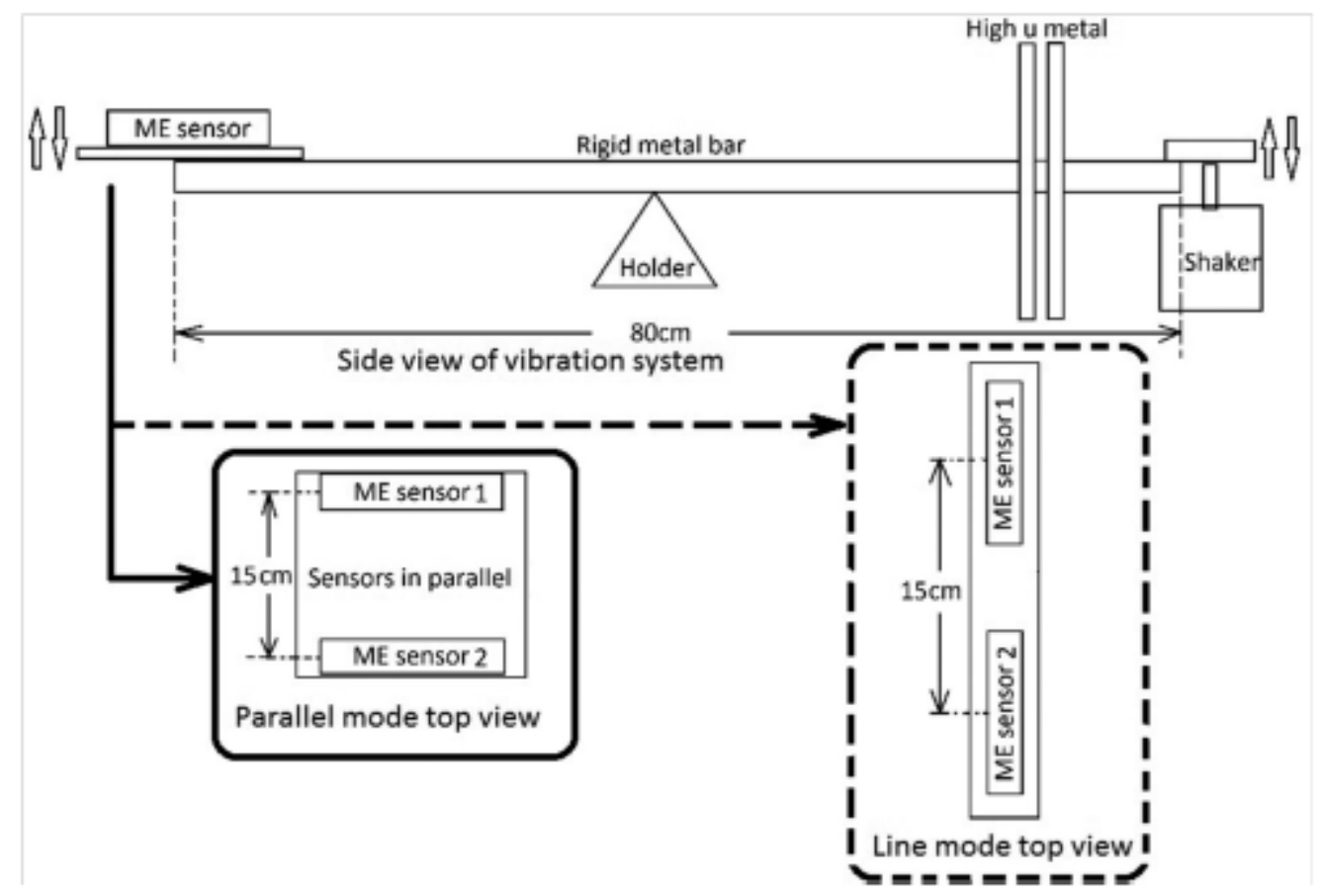

FIG. 13. Vibration rejection measurements of the ME gradiometer based on modulation techniques [70]. Reproduced from Journal of Applied Physics, 123, 104501 (2018), with the permission of AIP Publishing.

\section{$\underline{\text { Recent progress in magnetoelectric energy and power conversion }}$}

Energy converters (transducers) are devices which convert one form of energy to another. Energy harvesting is a process that collects/captures the waste energy in an environment (e.g. solar, thermal, mechanical, magnetic and/or electric fields.) and stores it for future usage. A 
present interest for collecting/storing energy by harvesting is for powering wireless sensor networks. Typical power consumptions of wireless sensor networks are on the range of micro to milliwatts. Otherwise, wired-powering methods are necessary. ME composites are good energy harvesters in the range of $n \mathrm{~W}$ to $\mu \mathrm{W}$ range. They can convert energy between magnetic and electrical forms, such as that required to harvest radiated magnetic energy generated by power carrying current cables.

Power conversion can be an electrical, electromechanical, or electromagnetic process for changing the characteristic of electrical energy, such as voltage to current or AC to DC. Conversion can be step-up or step-down. It can be achieved by a rectifier, transformer, voltage/current regulator, and/or gyrator, amongst others possibilities. ME composites combined with copper coils have been demonstrated to be a step-up/down power converter, and more recently as a gyrator that converters current to voltage, or conversely.

In the following paragraphs, ME-based energy harvesting and conversion devices will be discussed. Discussions of ME-based energy harvesters will be divided into two parts: vibration and magnetic. Discussions of power conversion will follow on transformers and gyrators.

\section{$\underline{\text { ME-based Vibration Energy Harvesters }}$}

The first conceptual approach that demonstrated the ME laminates ability to be an energy harvester was reported in 2003 by Huang et al. [77]. They proposed that a bipolar magnet changed the direction that flux coupled to the magnetostrictive layers of a ME composite. This was due to vibrations. The relative position between the ME composite and permanent magnet was changed by the external vibration that was incident upon it, resulting in an electrical voltage/power, via the direct ME effect. This energy harvester produced a power of $1 \mathrm{~mW}$ under a vibrational acceleration of $2 \mathrm{~g}$ at $45 \mathrm{~Hz}$. These results indicated that ME composites could be configured to harvest energy from mechanical vibrations. Numerous investigations of MEbased vibration energy harvesting were then reported. A one-directional bending mode vibration energy harvester was proposed based on tri-layer Terfenol-D/PZT/Terfenol-D ME laminates, which was configured as cantilever beams [78]. The design was based on a piezoelectric cantilever vibration energy harvester driven in a motion by bonded magnets at the end of the cantilever coupling to changes in the magnetic flux. Four NdFeB magnets were alternatively arranged by their poles forming a magnetic yoke. The harvester delivered a 
maximum power output of $1.01 \mathrm{~mW}$ at a resonance frequency of $51 \mathrm{~Hz}$ under an acceleration of $1 \mathrm{~g}$. Several years later, a similar architecture that employed multiple Terfenol-D/PMNPT/Terfenol-D tri-layer ME laminates with the same configuration of $\mathrm{NdFeB}$ magnets was proposed [79]. The maximum output power before saturation was $1.44 \mathrm{~mW}$ at an acceleration of $1 \mathrm{~g}$. However, the harvester produced $7.13 \mathrm{~mW}$ at $2.5 \mathrm{~g}$. Both structures were one directional cantilever vibrational energy harvesters.

The cantilever geometry is the most used structure for mechanical energy harvesting of vibrations, basically because of the simple assembly of the structure. However, importantly, a single resonance frequency of the fundamental flexural mode limited the bandwidth for harvesting. Accordingly, a recent trend has been to widen the bandwidth. Therefore, modification using multiple ME transducers to expand the bandwidth have been reported by Yang et al. [80] Three cantilever beams, two magnetoelectric laminates, and an alternating $\mathrm{NdFeB}$ magnet attached at the free end of the beam were combined to create three natural resonant frequencies, offering an effective bandwidth to the harvester of $7.2 \mathrm{~Hz}$ over a resonance range extending from 24 to $20 \mathrm{~Hz}$. It delivered a power of $0.21 \mathrm{~mW}$ under an acceleration of 0.2 g. In 2013, Bai et al. [81] reported a multi-modal ME vibration energy harvester based on a spiral-shaped cantilever. The spiral cantilever acted as a vibration resonator. Four magnets acted as a proof mass, bonded at the innermost layer of the cantilever. A ME laminate having a (longitudinal-transverse) $L-T$ configuration consisting of two Terfenol-D plates and a single PZT-5H one was fixed at different heights and placed in the middle of the resonator. Under vibration excitation, the spiral cantilever operated in bending and torsional modes, causing the magnets to move relative to the ME laminate. Motion thus occurred in multiple directions producing a complex magnetic field variation. Maximum output powers of 118.3, 25.1 80.5, 40.5, and 6.21 $\mu \mathrm{W}$ were found at different frequencies over the range of 15$70 \mathrm{~Hz}$ under an acceleration of 0.2g. In 2016, Lin at al. [82] proposed a similar energy harvester, which consisted of a folded cantilever, a ME laminate, and two magnetic circuits. Three ME laminates configured in a $L-T$ mode were arranged and positioned at the tip mass of the cantilever. The harvester was then tested at three different positions, and the results yielded a maximum average power of $31.6 \mu \mathrm{W}$ at an acceleration of $0.6 \mathrm{~g}$ at a frequency of $13 \mathrm{~Hz}$. Voltage peaks appeared at $13 \mathrm{~Hz}, 17.4 \mathrm{~Hz}$, and $22.9 \mathrm{~Hz}$, resulting in a working bandwidth of $10.1 \mathrm{~Hz}$.

Most energy harvesting devices collect power in a unidirectional mode. They lack the ability to harvest vibrations from other directions. To overcome this limitation, bi-axial ME vibration energy harvesters have been developed that utilize multiple cantilevers to extract 
vibration energy from multiple directions. In 2012, Yang et al. [83] proposed a harvester that consisted of two cantilevers and two ME laminates. The ME laminates were attached at the end of the two cantilevers as tip masses and consisted of a $L-T$ structure of Terfenol-D bonded to PMN-PT. These two cantilevers were then mounted along vertical directions and placed inside a four-alternatively poled permeant magnet configuration. The energy harvester had bandwidths of $2.8 \mathrm{~Hz}$ and $2.5 \mathrm{~Hz}$ along vertical and horizontal directions under a vibration acceleration of $0.6 \mathrm{~g}$, and the maximum output voltage was $130 \mathrm{~V}$. In the same year, Moss et al. [84] proposed a bi-axial ME energy harvester using a permanent-magnet/ball bearing arrangement, together with a ME laminate composite. The ME laminate was formed by bonding two Terfenol-D plates and a PZT layer into a $L-T$ mode structure. The magnetic flux distribution in the ME composite was changed when the ball bearing oscillated with a restoring force on the ball, and when it was free to move on the surface of the upper magnetostrictive layer. Under an external acceleration vibration of $61 \mathrm{mg}$ at $9.8 \mathrm{~Hz}$, a maximum power output of $121 \mu \mathrm{W}$ was achieved. Although the harvested power was relatively small, the vibration region of the ball bearing was relatively free to move, enabling the harvester to collect vibration energy in multiple directions.

Bandwidth and multi-directionality are two of the most important issues in the development of vibration energy harvesting. All previous designs could not overcome both these limitations at the same time. In 2016. Lin et al. [85] reported a 3-directional ME harvester consisting of a cylindrical magnet mounted by three springs, three ME laminates, and a mounting frame. The three springs and ME laminates were mounted symmetrically on the frame at an angle of $120^{\circ}$, and the angle between the springs and laminates was $60^{\circ}$. Under external vibrations, the relative motions of the center cylindrical magnet produced various magnetic field variations in arbitrary directions, enabling the direct ME effect of the ME laminate to harvest an electrical power. Different out-of-plane and in-plane vibration experiments were tested at different angles to confirm the harvesting ability in multi-directions. Experiments showed that a maximum load power of $12.9 \mu \mathrm{W}$ could be achieved over a wide bandwidth of $3.2 \mathrm{~Hz}$ at a natural resonance frequency of $10 \mathrm{~Hz}$ and under an acceleration of $1 \mathrm{~g}$. The schematic diagram of discussed multi-directional vibration-typed ME energy harvester is shown in Fig. 14. 


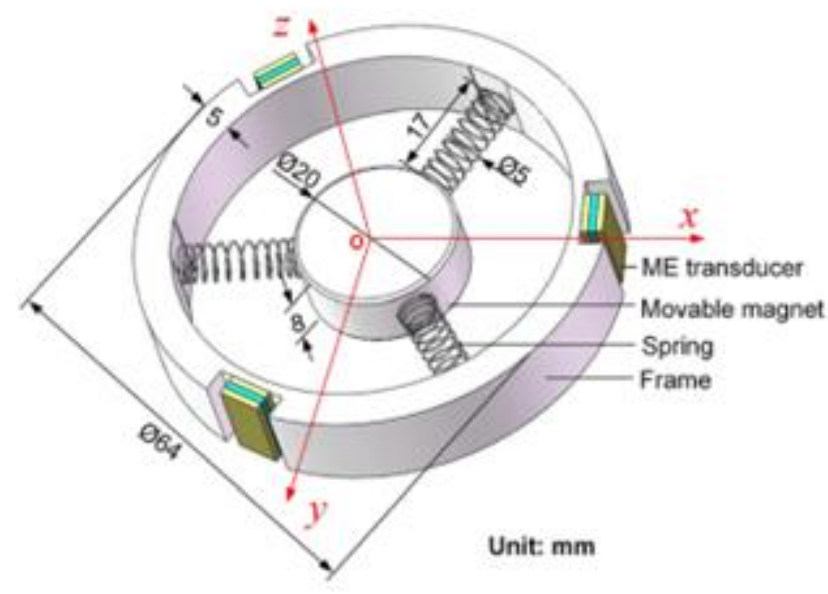

Fig.14. A central cylindrical magnet mounted by three identical springs inside a ring frame which attached with three ME transducers located in a $\mathbf{1 2 0}^{\mathbf{0}}$ arrangement [85]. Reproduced from Applied Physics Letters, 109, 253903 (2016), with the permission of AIP Publishing.

In recent years, researchers have put much effort into different designs to enhance the energy harvesting performance of ME composites. In 2012, Zhu et al. [86] proposed an energy harvester based on $L-T$ laminates, which was fixed to the surface of a center pole. Two magnets were fixed at the top and bottom of the pipe's outer surface, allowing the middle magnet to be free to move and levitate. Experiments showed a maximum power output of $1.1 \mathrm{~mW}$ under a vibration acceleration of $1 \mathrm{~g}$ at $10 \mathrm{~Hz}$. In 2013, Li et al. [87] reported a passive self-tuning energy harvester for collecting energy in rotating applications. The mechanical energy harvester had two hollow magnets with ME laminates placed inside the holes, and two cylindrical magnets mounted on the free end of a cantilever beam that was placed between the two free moving hollow magnets. The core ME laminate was formed by two Terfenol-D and one PMNPT plates with a $L-T$ configuration. By changing the distance between the magnets on the cantilever beam and the ME laminate, the power, voltage, vibration displacement, and frequency could all be tuned. The harvester was then mounted on a wheel driven by an ac servoactuator. Experiments found that a maximum power of $517 \mu \mathrm{W}$ could be harvested at a rotation frequency of $9.8 \mathrm{~Hz}$. Recently, in 2016, Dai et al. [88] proposed an energy harvester composed of a $L-T$ ME laminate and a rotary pendulum embedded with six magnets. The rotary pendulum was mounted on a supporting fixture using a bearing, and the ME laminate was placed between the magnets. This prototype produced a $3 \mathrm{~dB}$ bandwidth of $3.2 \mathrm{~Hz}$ and an output power of 970.2 $\mu \mathrm{W}$ under an acceleration of $0.5 \mathrm{~g}$ at $14.8 \mathrm{~Hz}$. 


\section{$\underline{\text { ME-based magnetic harvesters }}$}

Magnetic field or electric power line energy harvesting is an alternative use for the ME effect, such as the radiated magnetic energy generated from a power/current cord or line. To harvest this magnetic energy, in 2004, Bayrashev et al. [89] developed a disk-shaped sandwich ME laminate constructed by a PZT layer sandwiched between two Terfenol-D ones. This laminate was then placed near a permanent magnet that was attached to the shaft of a linear variable frequency $(1-30 \mathrm{~Hz})$ motor. The resulting output power was found to be $10-80 \mu \mathrm{W}$, depending on the distance between the ME laminate and permanent magnet.

In 2010, Wang et al. [90] characterized the effect of an electrical resistance load on the ME coupling using a $L-T$ mode Terfenol-D/PMN-PT/Terfenol-D ME laminate. The output power initially increased with increasing resistance load at resonance, reached a maximum value, and then decreased with further increase of resistance load. This observation was similar to that for systems using PZT type ME laminates connected with various load resistances. The results demonstrated that the proposed laminate was capable of producing $1.9 \mathrm{~mW}$ at a resonance frequency of $83.6 \mathrm{kHz}$.

One of the biggest challenges for practical application of ME laminates has been their relatively high resonance frequency which limits their application in energy harvesting from industrial/household electrical devices. In the real world, the frequencies of the electric power delivered are in the range of 50/60 Hz. Thus, it is highly desirable to tune the resonance frequency to considerably lower frequency ranges, in order to optimize the output power from ME harvesters. In 2012, Gao et al. [91] demonstrated a tunable unsymmetrical bi-layer Metglas/PZT ME composite that harvested $60 \mathrm{~Hz}$ electromagnetic energy. By attaching different numbers of permanent magnets as tip masses, the resonance frequency could be adjusted from 60 to $220 \mathrm{~Hz}$. The optimized output power of this harvester reached $16 \mu \mathrm{W} / \mathrm{Oe}$ at a resonance frequency of $60 \mathrm{~Hz}$ after tuning the tip mass. This demonstrates a simple and direct way to use ME laminates to harvest the magnetic energy generated by current carry cables. In the same year, Qiu et al. [92] reported a design for energy harvesters based on a ME bending configuration, allowing for significant reduction in device size. It consisted of cantilever beams, ME transducer, and a magnetic yoke configured by permeant magnets. A PZT -plate was bonded to the cantilever beam and fixed on a holder. An electric cable was placed in the center of the magnetic yoke and fixed to a support. When a current passed through the electric cable, an alternating force was generated via Ampere's law between current and magnetic field. Since 
the cable line was fixed to the holder, this force acted on the cantilever, causing it to vibrate and deform. An output power of $240 \mu \mathrm{W}$ was generated in response to an input current of $3 \mathrm{~A}$ at $50 \mathrm{~Hz}$.

A Halbach magnet array is a special arrangement of permanent magnets that enhances the magnetic field on one side of an array. A Halbach magnet mounted on the end of a cantilever has been employed with an $L-T$ mode Terfenol-D/PZT/Terfenol-D ME laminate to enhance the harvested power [93]. The results showed an output power of $523 \mu \mathrm{W}$ under an electric current of $5 \mathrm{~A}$ at $50 \mathrm{~Hz}$. Commonly two wires (live and neutral) are combined in a cord that is required to carry power to an appliance. The two wires are bundled parallel and placed close together, then a magnetic field from one will near-exactly cancel that from the other. Accordingly, a special design is required to overcome this limitation and to enhance the performance. In 2006, Leland et al. [94] proposed an energy harvesting device constructed from cantilever-mounted piezoelectric bimorphs and permanent magnets. It was able to harvest the radiated magnetic power from two such conductor power cables. A maximum power output of $208 \mu \mathrm{W}$ under 9.4A and $345 \mu \mathrm{W}$ under $13 \mathrm{~A}(60 \mathrm{~Hz})$ was found. In 2014, He et al [95] fabricated a similar bending mode ME harvester, designed for two-wire power cords. This energy harvester had a magnetic circuit consisting of six alternately poled $\mathrm{NdFeB}$ magnets mounted on the free end of a cantilever beam and a $L-T$ trilayer structure of Terfenol-D/PMN-PT/Terfenol-D. Because of the opposite arrangement of magnets, the Ampere forces acting on the two conductors were superimposed. This resulted in an enhanced maximum power of $671.2 \mu \mathrm{W}$ under a current of $6 \mathrm{~A}$ at $50 \mathrm{~Hz}$. All schematic diagrams, photos, and resulted output power of previous ME-based magnetic harvesters are shown in Fig. 15.

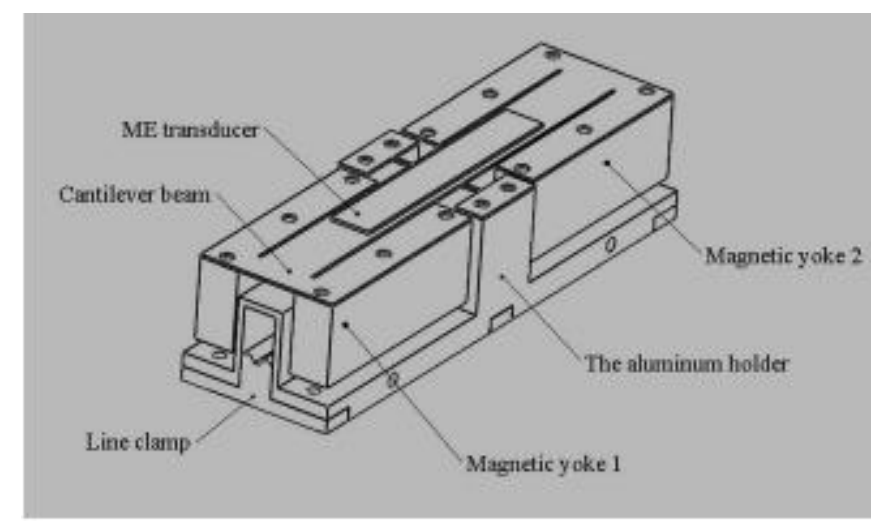

Fig. 15. Schematic diagram of the piezoelectric cantilever beam and ME transducer based magnetic fieldcurrent energy harvester for single core power cable [92]. Reproduced from Journal of Applied Physics, 111, 07E510 (2012), with the permission of AIP Publishing. 

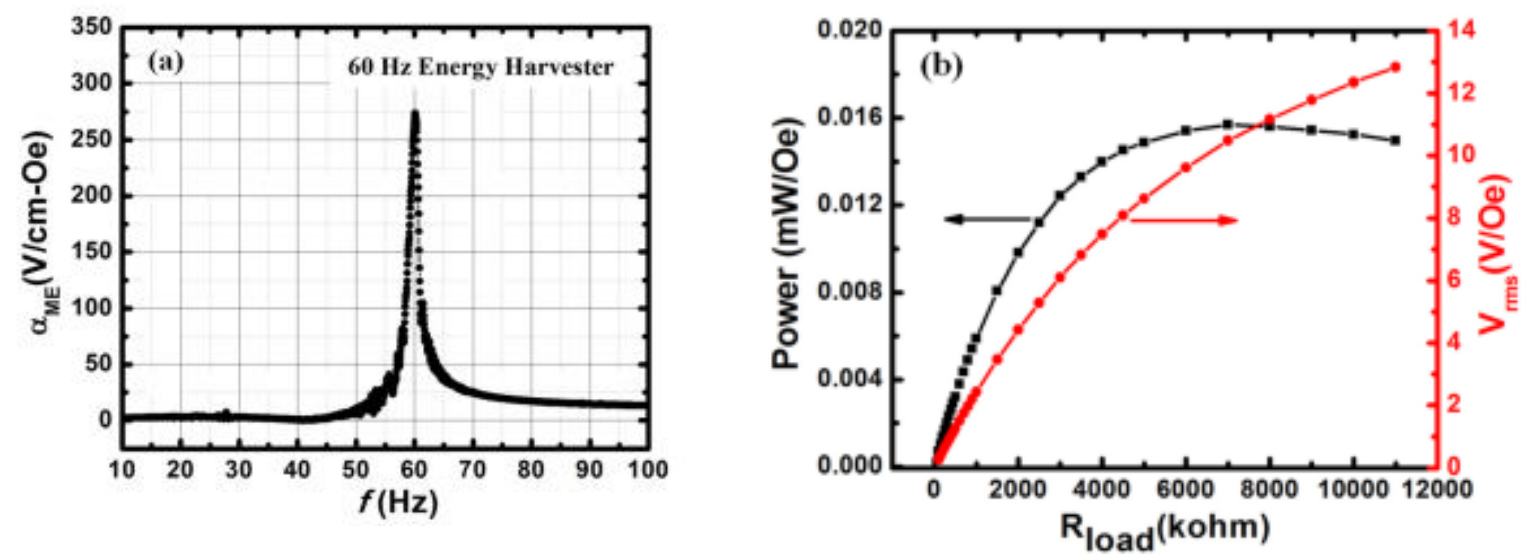

Fig. 16. (a) ME voltage coefficient of $60 \mathrm{~Hz}$ magnetic energy harvester as a function of AC magnetic drive frequency and (b) output voltage and power as a function of resistance load at the bending mode resonance frequency [91]. Reproduced from Journal of Applied Physics, 112, 104101 (2012), with the permission of AIP Publishing.

Unused power, which can be harvested from industrial machine/transportation vehicles is usually available as either vibration or magnetic fields. To enhance the power harvesting density, multimodal ME energy harvesters with various configurations that combined both vibration and magnetic harvesting mechanism were proposed by Dong at al. [96] in 2008. A ME laminate can be configured to harvest vibration and magnetic energies at the same time using the direct ME and direct piezoelectric effects. This was achieved by configuring the ME laminate from two push-pull symmetrically poled PZT fibers laminated together with four FeBSiC ribbons as shown in Fig. 17. The first longitudinal resonance frequency of the ME laminate was $21 \mathrm{kHz}$ without any mechanical vibration input, and the output power was $520 \mu \mathrm{W}$ under 1 Oe. However, in an open ambient environment, there are limited sources of emitted magnetic flux in this $20 \mathrm{kHz}$ frequency range. A cantilever beam structure with a tip mass was thus used to lower the frequency range to between 20 and $40 \mathrm{~Hz}$. A peak-to-peak output voltage of $8 \mathrm{~V}$ was obtained from both piezoelectric layers under a magnetic field of 2 Oe together with a vibration of $0.05 \mathrm{~g}$. This output voltage was doubled relative to a single power source, proving the possibility to harvest both vibration and magnetic field energies simultaneously. A flexible, low-cost energy-harvesting device based on the magnetoelectric (ME) effect was designed using $\mathrm{Fe}_{64} \mathrm{Co}_{17} \mathrm{Si}_{7} \mathrm{~B}_{12}$ as amorphous magnetostrictive ribbons and polyvinylidene fluoride (PVDF) as the piezoelectric element by Lasheras et al. [97] in 2015. A $3 \mathrm{~cm}-$ long sandwichtype laminated composite was fabricated by gluing the ribbons to the PVDF with an epoxy resin. A voltage multiplier circuit was designed to produce enough voltage to charge a battery. The power output and power density obtained were $6.4 \mu \mathrm{W}$ and $1.5 \mathrm{mWcm}^{-3}$, respectively, at optimum load resistance and measured at the magnetomechanical resonance of the laminate. 
(a)

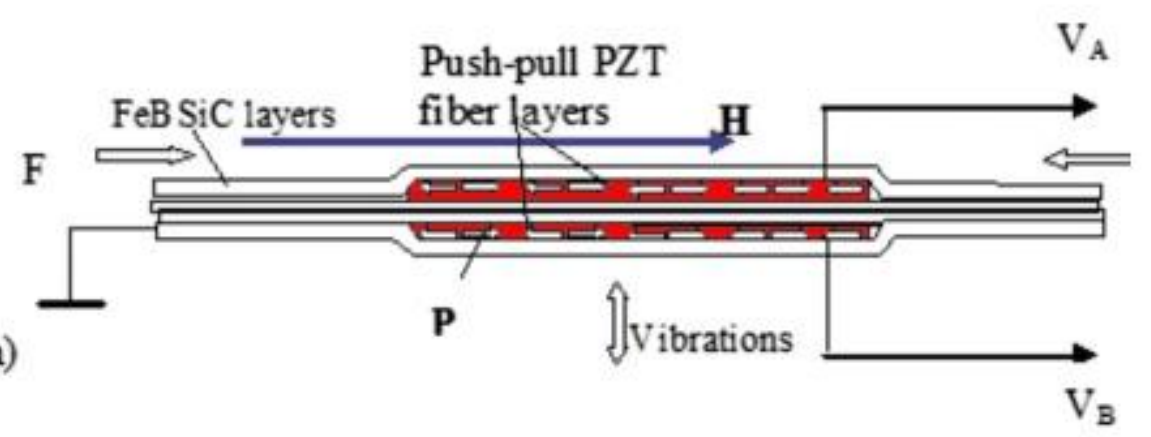

(b)

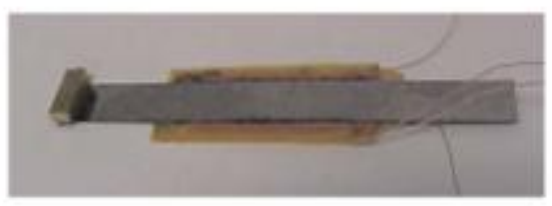

Fig. 17. Conceptual illustration of the ME energy harvester: (a) schematic of the ME laminate configuration with p polarization units and (b) photo of the ME laminate prototype, [96]. Reproduced from Applied Physics Letters, 93, 103511 (2008), with the permission of AIP Publishing.

Previous investigations have shown that the resonance frequency of ME beam harvesters can be reduced by changing the operational modes of the harvesting system. However, this additional mechanical load is also a damping term that reduces the efficiency of the harvester. To avoid this problem, in 2013, Zhou et al. [98] reported a dual-phase energy harvester. It was configured by combining a ME laminate and a piezoelectric unimorph bonded in a cantilever structure. The piezoelectric layer was a micro-fiber composite (MFC) bonded to a nickel cantilever beam. The schematic diagram self-bias dual-phase energy harvester is shown in Fig. 18. This cantilever not only served as a magnetostrictive phase to transfer the stress and strain to the piezoelectric layer, but also as a bender for vibrations to directly create strain in the piezoelectric layer. Under a vibration acceleration of $0.17 \mathrm{~g}$ at $22.5 \mathrm{~Hz}$, the harvester induced a maximum electrical power output of $168 \mu \mathrm{W}$. Under this hybrid approach (magnetic and vibration), they found that the vibration energy was the main contributing at a frequency of $22.5 \mathrm{~Hz}$. 


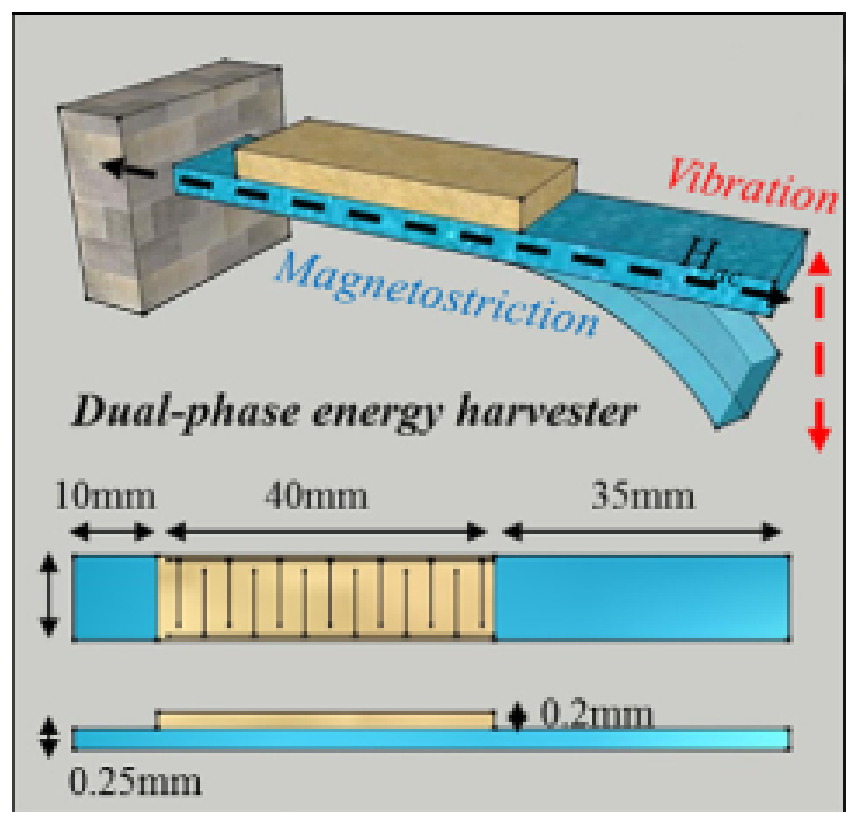

Fig.18. Schematic diagram of self-bias dual-phase energy harvester consisting of magnetostrictive bender and ME composite [98]. Reproduced from Applied Physics Letters, 103, 192909 (2013), with the permission of AIP Publishing.

In 2015, Qiu et al. [99] proposed a vibration-based energy harvester that hybrid ME and electromagnetic sources. It consisted of a magnetic circuit combined with four permanent magnets, a hybrid transducer combined with a coil-wrapped five-phase ME laminate and a cantilever. All components were fixed on an aluminum holder and mounted on a shaker for testing. The five-phase $\mathrm{ME}$ laminate was composed of $L-T \mathrm{FeCuNdSiB} / \mathrm{Terfenol-}$ D/PZT/Terfenol-D/FeCuNdSiB configuration. A maximum output power of $40.84 \mathrm{~mW}$ was found at a resonance frequency of $25.7 \mathrm{~Hz}$ under an acceleration of $0.75 \mathrm{~g}$.

\section{$\underline{\text { ME-based transformers/gyrators }}$}

Similar to a conventional magnetic transformer, ME laminates can be used as a stepup/down transformer, which transforms an ac voltage/current at the primary/input side to a proportional voltage/current at the secondary/output side. Such ME step-up or down converters have the potential to replace conventional transformers at their lower power levels, as they have smaller sizes and weight. In 2004, our team [100] observed a large gain effect in a ring-type ME laminate. It consisted of two circumferentially magnetized Terfenol-D rings and a circumferentially poled PZT one (consisting of 4 segments) wrapped with a toroidal coil. When an ac voltage was applied to the coil, an ac current flowed into the coil, subsequently creating a vortex magnetic field of the same frequency along the circumference direction of the laminate. Based on a direct ME effect under resonance drive, a higher voltage output was obtained in the 
piezoelectric layers. A maximum voltage gain of $25 \times$ was found under a magnetic bias of 500 Oe at $53.3 \mathrm{kHz}$. These findings demonstrated the feasibility that ME laminates could be used as a solid-state step-up power converter. In 2004, our team [101] found an extremely high voltage gain effect of $\approx 300$ in a rectangular-shaped ME laminate. The geometry was a long longitudinally poled push-pull piezoelectric layer sandwiched between two longitudinally magnetized Terfenol-D plates. By taking advantages of a high mechanical quality factor and the longitudinally poling of PZT, a maximum voltage gain of $260 \times$ was achieved at a resonance frequency of $21.3 \mathrm{kHz}$. A few years later, in 2009, Wang et al. [102] reported a large voltage gain of 130 at resonance using a long-type ME heterostructure consisted a length magnetized Terfenol-D layer and a length-polarized PMN-PT one. A detailed study of the voltage gain and output power from the converter as a function of resistive load confirmed that a similar load effect was observed as for piezoelectric transformers. The detailed structure of ME transformer configured in ring and sandwich were shown in Fig. 19. 
(a)

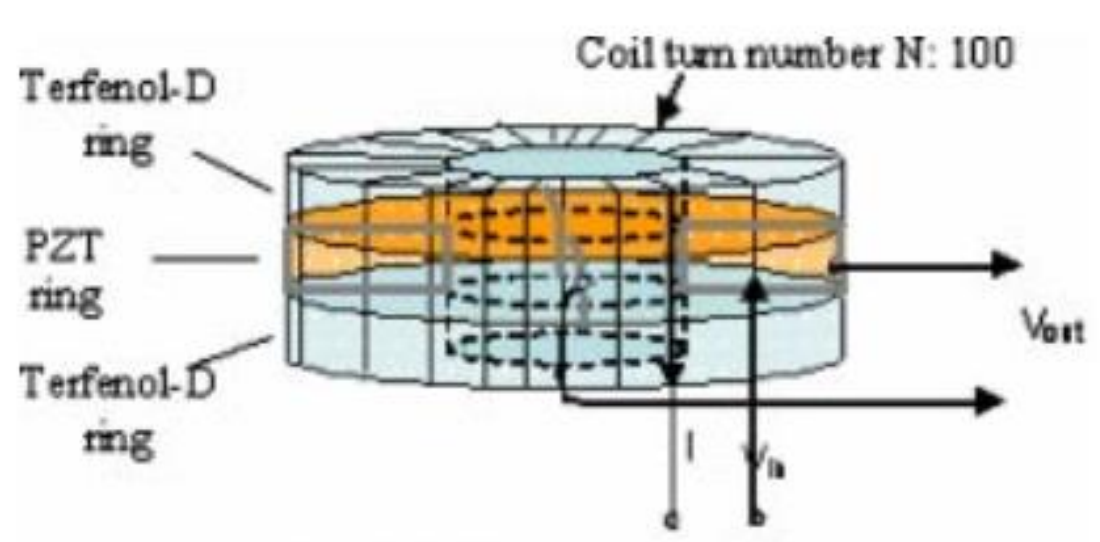

Voltage Gain $n=\mathrm{V}_{\text {out }} / V_{\text {in }}$

(b) Coil number: $\mathrm{N}$

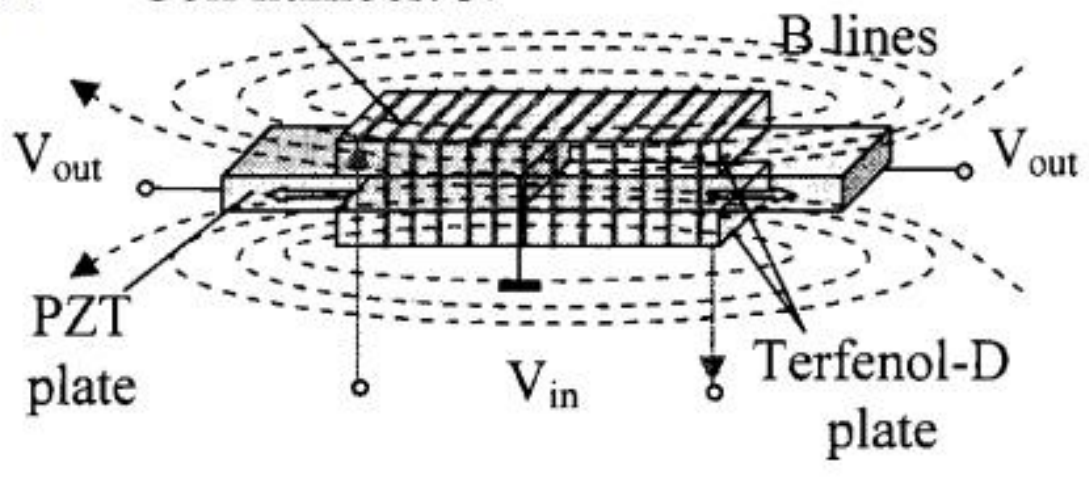

Fig.19. Different structures of ME transformer in (a) ring-type and (b) sandwich-type [100,101]. Reproduced from Applied Physics Letters, 84, 4188-90 (2004) and Applied Physics Letters, 85, 3534-6 (2004), with the permission of AIP Publishing.

Due to the resonance feature of ME-based laminates with a high-quality factor, they have potential applications as step-up/down converters in power electronics. The gain characteristics of ME converters are highly dependent on load and are useful only over a narrow frequency range near resonance. This has led to some challenges in design that require evaluation for a range of applications. Tunable ME gain effects and external magnetic fields have potential usefulness. In 2009, our team [18] made a tunable ME transformer that combined FeBSiC alloy ribbons and a piezoelectric PZT fiber operated in a push-pull mode. This laminate composite was wrapped by a copper coil that served as an input port. Because of the low magnetic bias field required for FeBSiC foils, a linear tunable voltage gain occurs under small magnetic bias changes between -5 and 5 Oe, where the maximum voltage gain of 55 was found under 5 Oe at resonance. In 2011, using the same DC magnetic field tunable properties in the magnetostrictive layer, Lv et al. [103] made a new type of ME transformer that combined a 
traditional Rosen-type piezoelectric transformer and a Terfenol-D plate. This allowed the voltage gain of the piezoelectric transformer to be controlled by dc magnetic bias fields. Two sharp peaks in the voltage gain were found. A maximum gain of 7.7 was found under zero magnetic bias that was enhanced to $9.2 \mathrm{~V}$ under 2500 Oe. Later, Zhou et al. [104] proposed a co-fired type ME transformer that consisted of two unipoled PZN-PT transformers and a nickelzinc copper (NZCF) ferrite layer (Fig. 20). This co-fired ME transformer exhibited a large frequency tunability effect. Although a voltage gain of only 1.52 was found at resonance without magnetic bias, a large frequency tunability of $1.4 \mathrm{~Hz} / \mathrm{Oe}$ was achieved under bias, opening up the possibility of developing on-chip converters for electronics.

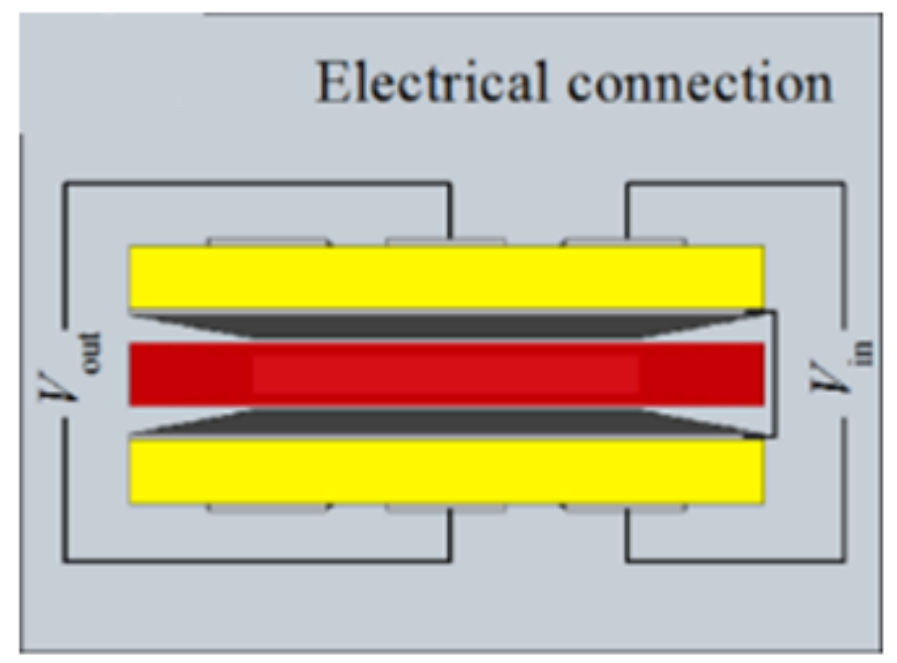

Fig. 20. Schematic diagrams of (a) co-fired PZNT/Ag/NCZF/Ag/PZNT laminate structure [104]. Reproduced from Applied Physics Letters, 104, 232906 (2013), with the permission of AIP Publishing.

Two-port four-wire ME laminates with lumped or distributed components may have applications such as transformers, waveguides, and antennas. The voltage to current relationship between inputs and outputs offer, for example, voltage-to-current conversions and maximum power transfer efficiency. Such network elements have characteristic expected of a gyrator. The gyrator was first conjectured by Tellegen [40] in 1948 to be a missing fifth network circuit element. It may be considered to be a two-port four-wire circuit which has the property that the phase shift for transmission in one direction differs by 180 degrees from that for transmission in the other direction. Another property is impedance inversion between the input and output ports, which provide unique current-to-voltage/voltage-to-current features. A conceptual diagram of ME gyrators was given in Fig.7. In 2006, our team [41] demonstrated that a $L-T$ Terfenol-D/PZT/Terfenol-D tri-layer laminates wrapped with a 50-turn copper wire 
had characteristics similar to gyrator properties. The investigation revealed a 180-degree phase shift at low frequencies, whose phase changed rapidly near resonance, and a large nondissipative $I-V$ conversation feature at resonance. In 2009, we reported a more detailed study of ME gyrators based on Terfenol-D/PZT/Terfenol-D, Metglas/PZT/Metglas, and $\mathrm{Ni} / \mathrm{PZT} / \mathrm{Ni}$ [105]. Experimental results showed that the Metglas based gyrators had superior power conversion ability, relative to Terfenol-D and Ni of similar dimensions [106]. These gyrators were configured with $L-T$ tri-layer structures. This study also reported conversion characteristics of capacitance to inductance, and impedance inversion. The voltage and current transfer relationships were discussed using the following impedance matrix given as [41]

$$
Z=\left[\begin{array}{ll}
Z_{11} & Z_{12} \\
Z_{21} & Z_{22}
\end{array}\right]=\left[\begin{array}{ll}
R_{11} & R_{12} \\
R_{21} & R_{22}
\end{array}\right]+j\left[\begin{array}{ll}
X_{11} & X_{12} \\
X_{21} & X_{22}
\end{array}\right]
$$

where $R$ and $X$ are the real and imaginary parts of the impedance. The ratio between the impedances $Z_{21}$ and $Z_{12}$ should be 1 , in order to guarantee an ideal gyrator. This leads to an equality of $\frac{\alpha_{M E}}{\sqrt{\varepsilon_{r} \mu_{r}}}=1$, where $\alpha_{\mathrm{ME}}$ is the magnetoelectric susceptivity and $\varepsilon_{r}$ and $\mu_{r}$ are the effective permittivity and permeability in ME composite.

In particular, the step-up/down $I-V / V$-I conversion features of ME laminates wrapped with a coil offers much potential as a ME gyrator. In 2009, our team [18] reported a ME transformer with tunable features using FeBSiC Metglas and hard PZT layers, which had an efficiency less than $50 \%$. The low efficiency was conjectured to be caused by various loss factors such as copper coil loss, Eddy current loss, dielectric loss and frictional loss imposed by the bonding layer (epoxy). A piezoelectric material with a high mechanical quality factor, Metglas foils with a higher resistivity, and an elastically matching bonding layer (different thickness ratios) have been suggested as approaches to improve the efficiency [18]. However, the power conversion characteristics of ME gyrators were not studied in detail, until 2016, when Leung et al. [107] reported the power conversion efficiency of a coil-ME gyrator based on Terfenol-D/PZT $L-T$ laminates. A detailed experimental setup is outlined in Fig. 21. The maximum values of the current-to-voltage coefficient was $1454 \mathrm{~V} / \mathrm{A}$ and the correspond voltage-to-current one was $0.468 \mathrm{~mA} / \mathrm{V}$, when operated at resonance. In this study, a power efficiency of $35 \%$ was found in both direct and converse modes. These findings showed that ME gyrators have power conversion characteristics that offer an approach to replace conventional electromagnetic and piezoelectric transformers. Such a low power efficiency of 
$35 \%$ would limit the application of ME gyrators as power converters. However, theoretical predictions indicated that ME composites should be capable of having power conversion efficiencies of greater than $90 \%$ [108].

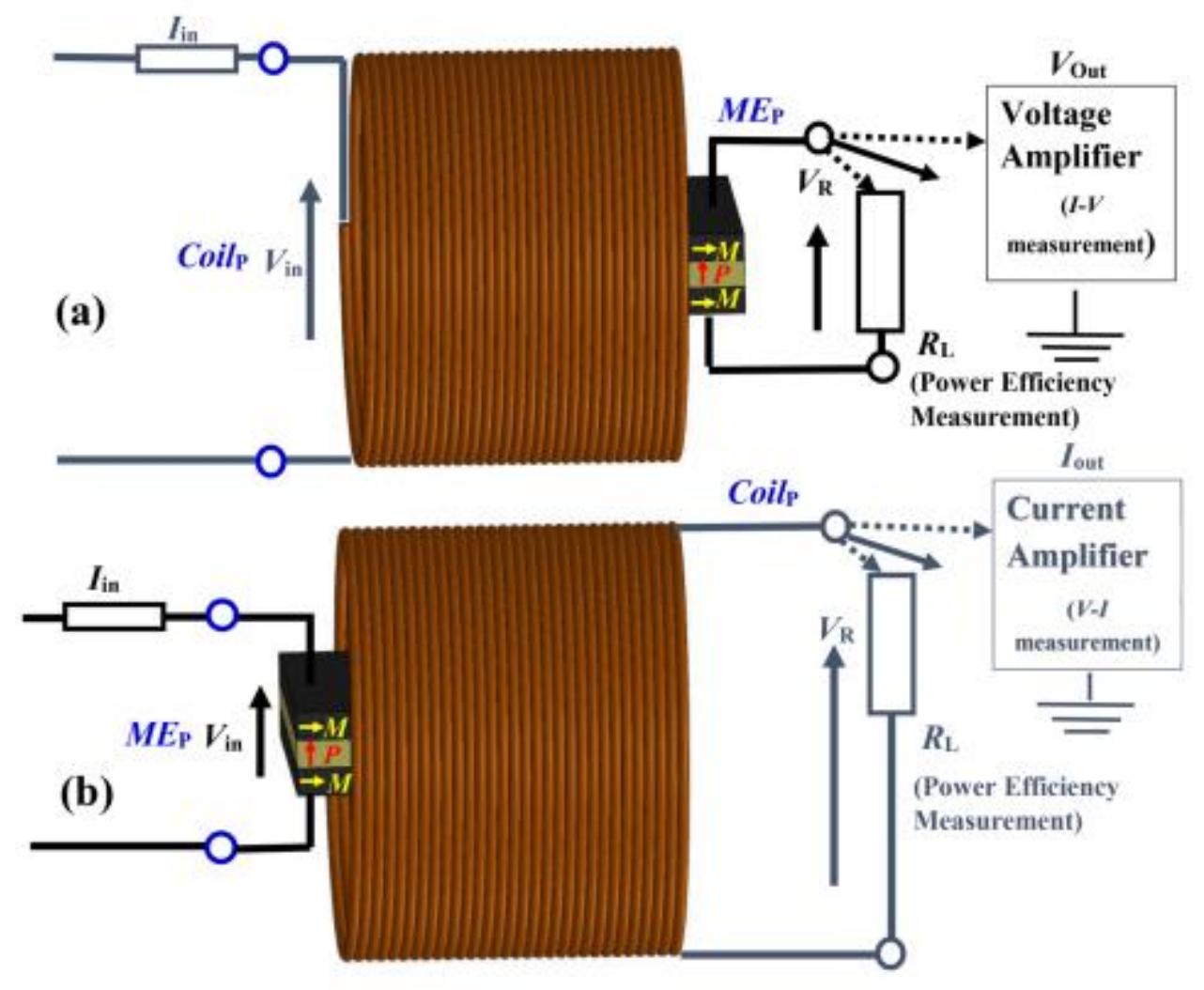

Fig.21. (a) $I-V$, (b) $V$-I, and (direct and converse) power efficiency measurement setup for ME gyrators [107]. Reproduced from Applied Physics Letters, 109, 202907 (2016), with the permission of AIP Publishing.

The path to improving the power efficiency $(\eta)$ of ME gyrators was to increase the product of the square of the effective coupling factor in the magnetostrictive phase $\left(k_{\text {eff, } m}\right)$ and the mechanical quality factor of the ME laminate $\left(Q_{\text {mech }}\right)$. Improvements in the power conversion efficiency of ME gyrators then focused on this important $k^{2}{ }_{\text {eff, } m} \cdot Q_{m e c h}$ product [109]. Experimental investigations have confirmed that both high $k_{e f f, m}$ and $Q_{m e c h}$ are important factors for increasing $\eta$. In 2017, Leung et al. [110] reported another important parameter that affected the power efficiencies of ME gyrators. For Terfenol-D/PZT/Terfenol-D tri-layers, the efficiency was found to be increased by decreasing the thickness/volume ratio between Terfenol-D and PZT phases. This is because the average mechanical quality factor was increased by decreasing the ratio of Terfenol-D in the ME laminate: $Q_{m e c h}$ of PZT was $\sim 500$, whereas that of Terfenol-D was $\sim 7$. Investigations showed that the volume fraction of the Terfenol-D magnetostrictive layer was the main limitation of $\eta$ for ME gyrators. NZFO was then proposed to replace the Terfenol-D layer in ME gyrators, in order to reduce the magnetic 
core loss due to Eddy currents. NZFO has a much small Eddy current loss, and also has a high magnetostriction [28].

Our research team then experimentally identified the loss mechanism of ME gyrators by using a transformer-gyrator structure [111]. At low power conditions, an optimal (but not matched) load resistor reflected a portion of the power. This was revealed as a decrease in the magnetomechanical conversion efficiency in the magnetostrictive phase. Under high power conditions, a portion of the power was transformed back to its pure magnetic form and captured as a leakage power by the secondary coil.

A high power density of $60 \mathrm{Watt} / \mathrm{in}^{3}$ has recently been achieved for a Metglas/PZT gyrator [111]. This makes ME gyrators promising devices for high power applications. $\eta$ was also increased to $85 \%$ for nickel-zinc ferrite/PZT ME gyrators at low power conditions $\left(\sim 20 \mathrm{~mW} / \mathrm{in}^{3}\right)$, and $\geq 80 \%$ at higher power conditions $\left(\sim 5 \mathrm{~W} / \mathrm{in}^{3}\right)$. This increase was due to the high mechanical quality factor of the ferrite [28]. Next, piezoelectric-magnetostrictivepiezoelectric (P-M-P) trilayer structures were designed. The P-M-P structure resulted in enhanced power efficiency stability for the ferrite-based gyrators with increasing power density, reaching an efficiency of $79 \%$ under $47 \mathrm{Watt} / \mathrm{in}^{3}$ [112]. The magnetomechanical conversion efficiency was directly measured as a function of power density [113], revealing that both Metglas and ferrites have extremely high magnetomechanical conversion efficiencies under low power conditions. However, it was found that Metglas can retain its superior efficiency at higher power drive conditions than ferrite. By using a piezoelectric material with a high mechanical quality factor, an optimal thickness ratio and a matching length of the coil to ME composite, a high power efficiency of greater than $90 \%$ was first achieved by Zhuang et al. [113]. 

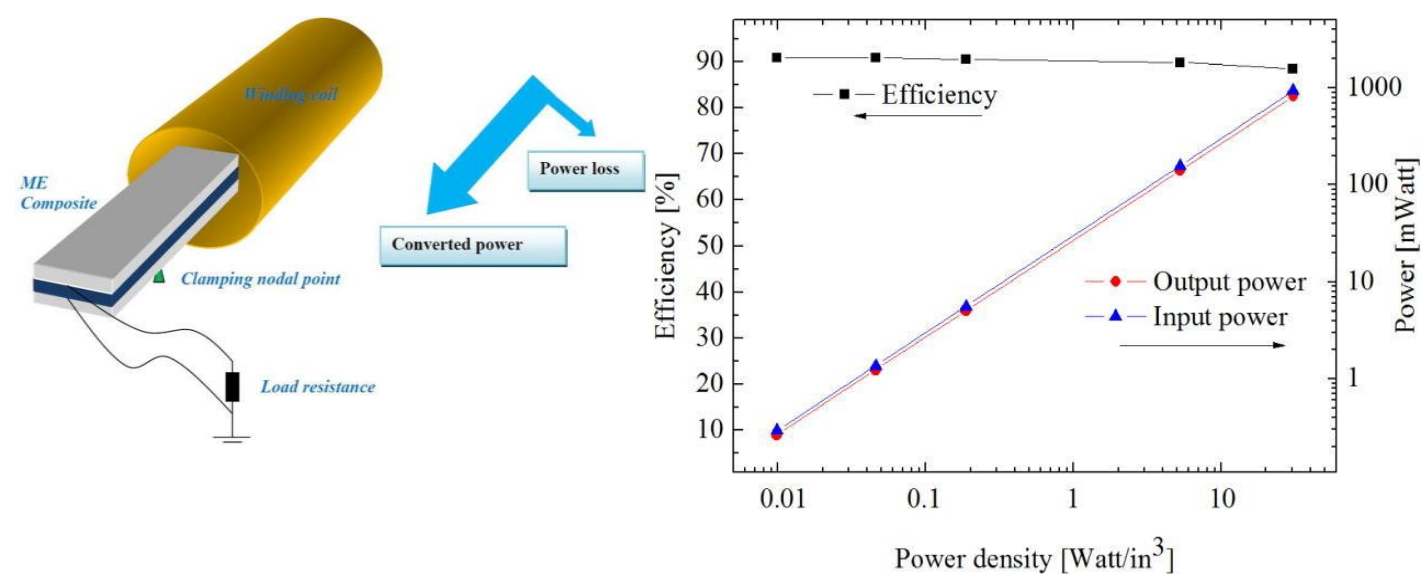

Fig. 22. Structure and power performance of Metglas/PZT gyrators [113]. Reproduced from Applied Physics Letters, 111, 163902 (2017), with the permission of AIP Publishing.

Under high drive conditions, the efficiency of Metglas/PZT gyrators have been found to retain their superiority $(\eta=89 \%)$ up to power densities of $30 \mathrm{Watt} / \mathrm{in}^{3}$, as shown in FIG. 23 , offering the potential to realize ME gyrators in applications as power electronic devices. The Metglas used in this ME configuration was annealed resulting in a minor volume fraction of $\mathrm{Fe}$ nano-crystallites, and it also contained manganese and carbon dopants (FeCoSiBMnC). Both the Metglas and PZT phases were hardened to achieve extraordinarily high mechanical quality factors of $\sim 1000$. Figure 28 summarizes a list of the significant investigations of power density and power efficiency for ME gyrators with different material combinations in Refs $[18,107,110,111,28,113,112]$, respectively.

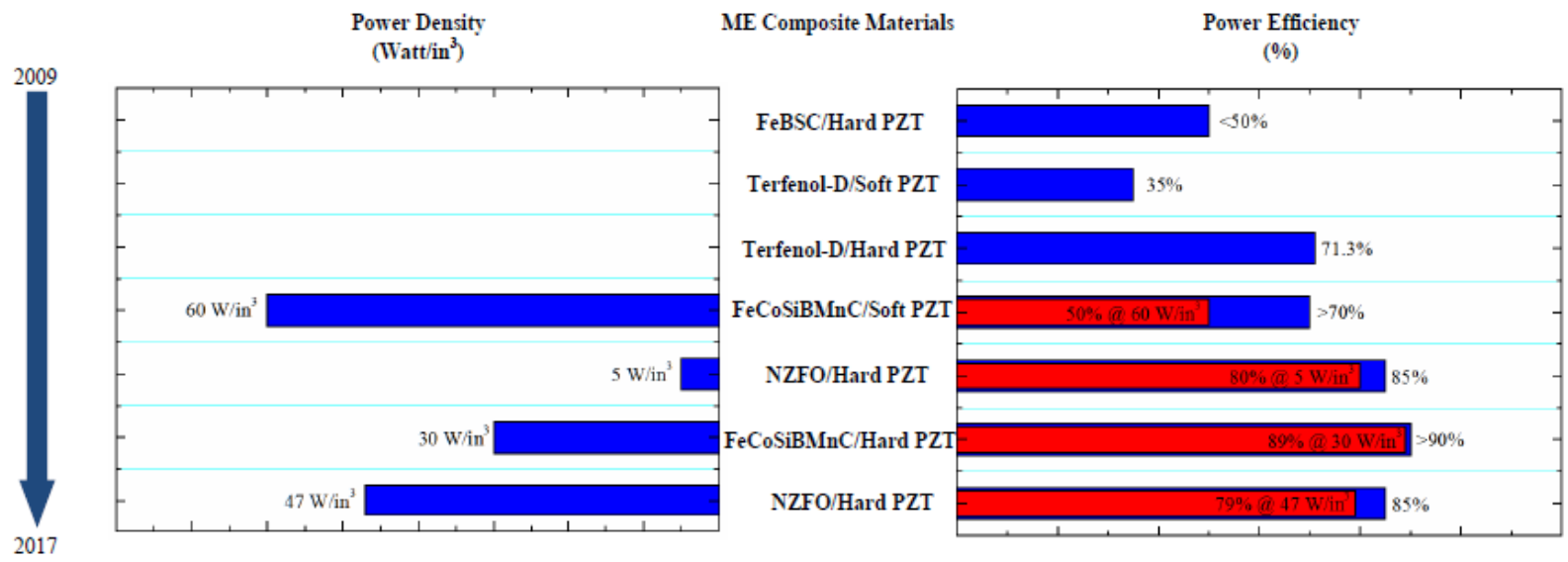

Fig. 23. Reported maximum values of power density and power efficiency for various type materials in Refs $[18,107,110,111,28,113,112]$, respectively. 


\section{Conclusion}

The focus of this review was on recent progress in ME composites and their applications from low to high power conditions. It is difficult to cover all ME materials that have recently been developed. Recent research activities on macro ME composites was discussed. Many investigations have advanced these materials towards real-world applications. These applications include magnetic sensors, energy harvesters, and power converters. Starting with a brief summary of the history of the ME effect, the paper then focused on the recent development of macro ME composites and their applications, as well their figures of merit. Non-resonant and resonant enhancements of the ME coefficients were discussed for various magnetostrictive (e.g. ferrites, transition metals, and alloys) and piezoelectric phases. The development of the ME sensors based on the selection of material properties and fabrication methods have been discussed, which resulted in a new type of magnetic sensors with minute power consumption and extremely high detection performance, which are competitive to existing magnetic sensor. Different ME-based energy harvesters have been compared according to their directionality, bandwidth, and sources of energy. Finally, a summary of recent advances in gyrators based on ME composites has been included in this review. The voltage/current stepup/down ratios and the power performances, involving power efficiency and power density, have been presented and discussed, which offer the ME gyrator as a potential electronic device for power conversion applications. We believe that ME composites offer the potential to unleash applications of non-destructive detections [114], power controlling systems in smart grids [115], wireless power transmission [116], and communications [117]. This promising potential is enabled by the high ME coupling that is unique to ME laminates and their devices.

\section{Acknowledgements}

This work was supported by the Defense Advanced Research Projects Agency through the MATRIX Program, grant number W911NF-15-1-0616. 


\section{Reference}

[1] Röntgen W C 1888 Ueber die durch Bewegung eines im homogenen electrischen Felde befindlichen Dielectricums hervorgerufene electrodynamische Kraft Ann. Phys. $271264-70$

[2] Curie P 1894 Sur la symétrie dans les phénomènes physiques, symétrie d'un champ électrique et d'un champ magnétique J. Phys. Théorique Appliquée 3 393-415

[3] Debye P 1926 Bemerkung zu einigen neuen Versuchen über einen magnetoelektrischen Richteffekt Z. Für Phys. 36 300-1

[4] Astrov D N 1960 The magnetoelectric effect in antiferromagnetics Sov Phys JETP 11 708-9

[5] Astrov D N 1961 Magnetoelectric effect in chromium oxide Sov Phys JETP 13 729-33

[6] Fiebig M 2005 Revival of the magnetoelectric effect J. Phys. Appl. Phys. 38 R123

[7] Ma J, Hu J, Li Z and Nan C-W 2011 Recent Progress in Multiferroic Magnetoelectric Composites: from Bulk to Thin Films Adv. Mater. 23 1062-87

[8] Eerenstein W, Mathur N D and Scott J F 2006 Multiferroic and magnetoelectric materials Nature $\mathbf{4 4 2} 759$

[9] Nan C-W, Bichurin M I, Dong S, Viehland D and Srinivasan G 2008 Multiferroic magnetoelectric composites: Historical perspective, status, and future directions $J$. Appl. Phys. 103031101

[10] Velev J P, Jaswal S S and Tsymbal E Y 2011 Multi-ferroic and magnetoelectric materials and interfaces Philos. Trans. R. Soc. Lond. Math. Phys. Eng. Sci. 3693069 97

[11] Van Suchtelen J 1972 Product properties: a new application of composite materials Philips Res Rep 27 28-37

[12] Boomgaard J V D, Terrell D R, Born R a. J and Giller H F J I 1974 An in situ grown eutectic magnetoelectric composite material J. Mater. Sci. 9 1705-9

[13] Ryu J, Carazo A V, Uchino K and Kim H-E 2001 Magnetoelectric Properties in Piezoelectric and Magnetostrictive Laminate Composites Jpn. J. Appl. Phys. 404948

[14] Dong S, Li J-F and Viehland D 2003 Longitudinal and transverse magnetoelectric voltage coefficients of magnetostrictive/piezoelectric laminate composite: theory IEEE Trans. Ultrason. Ferroelectr. Freq. Control 50 1253-61

[15] Dong S, Li J-F and Viehland D 2004 Longitudinal and transverse magnetoelectric voltage coefficients of magnetostrictive/ piezoelectric laminate composite: experiments IEEE Trans. Ultrason. Ferroelectr. Freq. Control 51 794-9

[16] Zhai J, Dong S, Xing Z, Li J and Viehland D 2006 Giant magnetoelectric effect in Metglas/polyvinylidene-fluoride laminates Appl. Phys. Lett. 89083507 
[17] Dong S, Zhai J, Li J and Viehland D 2006 Near-ideal magnetoelectricity in highpermeability magnetostrictive/piezofiber laminates with a (2-1) connectivity Appl. Phys. Lett. 89252904

[18] Dong S, Zhai J, Priya S, Li J F and Viehland D 2009 Tunable features of magnetoelectric transformers IEEE Trans. Ultrason. Ferroelectr. Freq. Control 56 $1124-7$

[19] Wang Y, Gray D, Berry D, Gao J, Li M, Li J and Viehland D 2011 An Extremely Low Equivalent Magnetic Noise Magnetoelectric Sensor Adv. Mater. 23 4111-4

[20] Gao J, Shen L, Wang Y, Gray D, Li J and Viehland D 2011 Enhanced sensitivity to direct current magnetic field changes in Metglas/ $\mathrm{Pb}(\mathrm{Mg} 1 / 3 \mathrm{Nb} 2 / 3) \mathrm{O} 3-\mathrm{PbTiO} 3$ laminates J. Appl. Phys. 109074507

[21] Lasheras A, Gutiérrez J and Barandiarán J M 2017 Size effects in the equivalent magnetic noise of layered Fe64Co17Si7B12/PVDF/Fe64Co17Si7B12 magnetoelectric sensors Sens. Actuators Phys. 263 488-92

[22] Wang Y, Gray D, Gao J, Berry D, Li M, Li J, Viehland D and Luo H 2012 Improvement of magnetoelectric properties in Metglas/ $\mathrm{Pb}(\mathrm{Mg} 1 / 3 \mathrm{Nb} 2 / 3) \mathrm{O} 3-\mathrm{PbTiO} 3$ laminates by poling optimization J. Alloys Compd. 519 1-3

[23] Kirchhof C, Krantz M, Teliban I, Jahns R, Marauska S, Wagner B, Knöchel R, Gerken M, Meyners D and Quandt E 2013 Giant magnetoelectric effect in vacuum Appl. Phys. Lett. 102232905

[24] Chu Z, Shi H, Shi W, Liu G, Wu J, Yang J and Dong S 2017 Enhanced Resonance Magnetoelectric Coupling in (1-1) Connectivity Composites Adv. Mater. 29 n/a-n/a

[25] Bichurin M I, Filippov D A, Petrov V M, Laletsin V M, Paddubnaya N and Srinivasan G 2003 Resonance magnetoelectric effects in layered magnetostrictive-piezoelectric composites Phys. Rev. B 68132408

[26] Gheevarughese V, Laletsin U, Petrov V M, Srinivasan G and Fedotov N A 2007 Lowfrequency and resonance magnetoelectric effects in lead zirconate titanate and singlecrystal nickel zinc ferrite bilayers J. Mater. Res. 22 2130-5

[27] Venkata Ramanaa M, Ramamanohar Reddy N, Sreenivasulu G, Siva kumar K V, Murty B S and Murthy V R K 2009 Enhanced mangnetoelectric voltage in multiferroic particulate $\mathrm{Ni} 0.83 \mathrm{Co} 0.15 \mathrm{Cu} 0.02 \mathrm{Fe} 1.9 \mathrm{O} 4-\delta / \mathrm{PbZr} 0.52 \mathrm{Ti} 0.48 \mathrm{O} 3$ composites - dielectric, piezoelectric and magnetic properties Curr. Appl. Phys. 9 1134-9

[28] Leung C M, Zhuang X, Friedrichs D, Li J, Erickson R W, Laletin V, Popov M, Srinivasan G and Viehland D 2017 Highly efficient solid state magnetoelectric gyrators Appl. Phys. Lett. 111122904

[29] Cho K-H, Yan Y, Folgar C and Priya S 2014 Zigzag-shaped piezoelectric based high performance magnetoelectric laminate composite Appl. Phys. Lett. 104222901 
[30] Wang Y, Gao J, Li M, Hasanyan D, Shen Y, Li J, Viehland D and Luo H 2012 Ultralow equivalent magnetic noise in a magnetoelectric Metglas/Mn-doped $\mathrm{Pb}(\mathrm{Mg} 1 / 3 \mathrm{Nb} 2 / 3) \mathrm{O} 3-\mathrm{PbTiO} 3$ heterostructure Appl. Phys. Lett. 101022903

[31] Sreenivasulu G, Petrov V M, Fetisov L Y, Fetisov Y K and Srinivasan G 2012 Magnetoelectric interactions in layered composites of piezoelectric quartz and magnetostrictive alloys Phys. Rev. B 86214405

[32] Sreenivasulu G, Fetisov L Y, Fetisov Y K and Srinivasan G 2012 Piezoelectric single crystal langatate and ferromagnetic composites: Studies on low-frequency and resonance magnetoelectric effects Appl. Phys. Lett. 100052901

[33] Jin J, Lu S-G, Chanthad C, Zhang Q, Haque M A and Wang Q 2011 Multiferroic Polymer Composites with Greatly Enhanced Magnetoelectric Effect under a Low Magnetic Bias Adv. Mater. 23 3853-8

[34] Freeman E, Harper J, Goel N, Gilbert I, Unguris J, Schiff S J and Tadigadapa S 2017 Improving the magnetoelectric performance of Metglas/PZT laminates by annealing in a magnetic field Smart Mater. Struct. 26085038

[35] Xin C, Ma J, Ma J and Nan C 2017 Stretch-shear mode laminated metglas/Pb (Zr, Ti) O-3/metglas composite with an enhanced magnetoelectric effect Sci. Bull. 6004

[36] Zeng L, Zhou M, Bi K and Lei M 2016 Giant magnetoelectric effect in negative magnetostrictive/piezoelectric/positive magnetostrictive semiring structure $J$. Appl. Phys. 119034102

[37] Zhang J, Kang Y, Yu Y and Gao Y 2017 Enhancement of the magnetoelectric coupling in an A-line shape magnetostrictive/piezoelectric structure Phys. Lett. A 381 $1-9$

[38] Hu J-M, Li Z, Wang J and Nan C W 2010 Electric-field control of strain-mediated magnetoelectric random access memory J. Appl. Phys. 107093912

[39] Hu J-M, Li Z, Wang J, Ma J, Lin Y H and Nan C W 2010 A simple bilayered magnetoelectric random access memory cell based on electric-field controllable domain structure J. Appl. Phys. 108043909

[40] Tellegen B D 1948 The gyrator, a new electric network element Philips Res Rep 3 81101

[41] Zhai J, Li J, Dong S, Viehland D and Bichurin M I 2006 A quasi(unidirectional) Tellegen gyrator J. Appl. Phys. 100124509

[42] Gao J, Gray D, Shen Y, Li J and Viehland D 2011 Enhanced dc magnetic field sensitivity by improved flux concentration in magnetoelectric laminates Appl. Phys. Lett. 99153502

[43] Shen L, Li M, Gao J, Shen Y, Li J F, Viehland D, Zhuang X, Lam Chok Sing M, Cordier C, Saez S and Dolabdjian C 2011 Magnetoelectric nonlinearity in magnetoelectric laminate sensors J. Appl. Phys. 110114510 
[44] Li M, Wang Z, Wang Y, Li J and Viehland D 2013 Giant magnetoelectric effect in self-biased laminates under zero magnetic field Appl. Phys. Lett. 102082404

[45] Zhuang X, Sing M L C, Cordier C, Saez S, Dolabdjian C, Das J, Gao J, Li J and Viehland D 2011 Analysis of Noise in Magnetoelectric Thin-Layer Composites Used as Magnetic Sensors IEEE Sens. J. 11 2183-8

[46] Kiser J, Lacomb R, Bussmann K, Hawley C J, Spanier J E, Zhuang X, Dolabdjian C, Lofland S and Finkel P 2014 Magnetostrictive stress reconfigurable thin film resonators for near direct current magnetoelectric sensors Appl. Phys. Lett. 104072408

[47] Nan T, Hui Y, Rinaldi M and Sun N X 2013 Self-Biased 215MHz Magnetoelectric NEMS Resonator for Ultra-Sensitive DC Magnetic Field Detection Sci. Rep. 31985

[48] Fetisov Y K, Serov V N, Fetisov L Y, Makovkin S A, Viehland D and Srinivasan G 2016 A magnetoelectric composite based signal generator Appl. Phys. Lett. 108213502

[49] Coblenz W S and Wartenberg S A 2012 The DARPA HUMS program: revolutionizing magnetic field sensors using multiferroic materials and atomic gas vapor cells Unattended Ground, Sea, and Air Sensor Technologies and Applications XIV vol 8388 (International Society for Optics and Photonics) p 838809

[50] Xing Z, Dong S, Zhai J, Yan L, Li J and Viehland D 2006 Resonant bending mode of Terfenol-D/steel $\mathrm{Pb}(\mathrm{Zr}, \mathrm{Ti}) \mathrm{O} 3$ magnetoelectric laminate composites Appl. Phys. Lett. 89 112911

[51] Dong S, Zhai J, Xing Z, Li J and Viehland D 2007 Giant magnetoelectric effect (under a dc magnetic bias of 2Oe) in laminate composites of FeBSiC alloy ribbons and $\mathrm{Pb}(\mathrm{Zn1} / 3, \mathrm{Nb} 2 / 3) \mathrm{O} 3-7 \% \mathrm{PbTiO} 3$ fibers Appl. Phys. Lett. 91022915

[52] Annapureddy V, Palneedi H, Yoon W-H, Park D-S, Choi J-J, Hahn B-D, Ahn C-W, Kim J-W, Jeong D-Y and Ryu J 2017 A pT/ $\sqrt{H z}$ sensitivity ac magnetic field sensor based on magnetoelectric composites using low-loss piezoelectric single crystals Sens. Actuators Phys. 260 206-11

[53] Zhuang X, Cordier C, Saez S, Lam Chok Sing M, Dolabdjian C, Gao J, Li J F and Viehland D 2011 Theoretical analysis of the intrinsic magnetic noise spectral density of magnetostrictive-piezoelectric laminated composites J. Appl. Phys. 109124512

[54] Gao J, Wang Z, Shen Y, Li M, Wang Y, Finkel P, Li J and Viehland D 2012 Selfpowered low noise magnetic sensor Mater. Lett. 82 178-80

[55] Bhaskaran H, Li M, Garcia-Sanchez D, Zhao P, Takeuchi I and Tang H X 2011 Active microcantilevers based on piezoresistive ferromagnetic thin films Appl. Phys. Lett. 98 013502

[56] Zhuang X, Sing M L C, Dolabdjian C, Wang Y, Finkel P, Li J and Viehland D 2015 Mechanical Noise Limit of a Strain-Coupled Magneto(Elasto)electric Sensor Operating Under a Magnetic or an Electric Field Modulation IEEE Sens. J. 15 1575-87 
[57] Mandal S K, Sreenivasulu G, Petrov V M and Srinivasan G 2010 Flexural deformation in a compositionally stepped ferrite and magnetoelectric effects in a composite with piezoelectrics Appl. Phys. Lett. 96192502

[58] Zhuang X, Saez S, Lam Chok Sing M, Cordier C, Dolabdjian C, Li J, Viehland D, Mandal S K, Sreenivasulu G and Srinivasan G 2012 Investigation on the Magnetic Noise of Stacked Magnetostricitive-Piezoelectric Laminated Composites Sens. Lett. 10 $961-5$

[59] Chen Y, Gillette S M, Fitchorov T, Jiang L, Hao H, Li J, Gao X, Geiler A, Vittoria C and Harris V G 2011 Quasi-one-dimensional miniature multiferroic magnetic field sensor with high sensitivity at zero bias field Appl. Phys. Lett. 99042505

[60] Srinivasan G, Rasmussen E T, Gallegos J, Srinivasan R, Bokhan Y I and Laletin V M 2001 Magnetoelectric bilayer and multilayer structures of magnetostrictive and piezoelectric oxides Phys. Rev. B 64214408

[61] Li M, Berry D, Das J, Gray D, Li J and Viehland D 2011 Enhanced Sensitivity and Reduced Noise Floor in Magnetoelectric Laminate Sensors by an Improved Lamination Process J. Am. Ceram. Soc. 94 3738-41

[62] Wang Y, Gray D, Berry D, Li M, Gao J, Li J and Viehland D 2012 Influence of interfacial bonding condition on magnetoelectric properties in piezofiber/Metglas heterostructures J. Alloys Compd. $\mathbf{5 1 3} 242-4$

[63] Filippov D A, Galichyan T A and Laletin V M 2014 Influence of an interlayer bonding on the magnetoelectric effect in the layered magnetostrictive-piezoelectric structure Appl. Phys. A 116 2167-71

[64] Quandt E, Stein S and Wuttig M 2005 Magnetic vector field sensor using magnetoelectric thin-film composites IEEE Trans. Magn. 41 3667-9

[65] Piorra A, Jahns R, Teliban I, Gugat J L, Gerken M, Knöchel R and Quandt E 2013 Magnetoelectric thin film composites with interdigital electrodes Appl. Phys. Lett. 103 032902

[66] Röbisch V, Salzer S, Urs N O, Reermann J, Yarar E, Piorra A, Kirchhof C, Lage E, Höft M, Schmidt G U, Knöchel R, McCord J, Quandt E and Meyners D 2017 Pushing the detection limit of thin film magnetoelectric heterostructures J. Mater. Res. 32 100919

[67] Yarar E, Salzer S, Hrkac V, Piorra A, Höft M, Knöchel R, Kienle L, and Quandt E 2016 Inverse bilayer magnetoelectric thin film sensor Appl. Phys. Lett. 109022901

[68] Xing Z, Zhai J, Li J and Viehland D 2009 Investigation of external noise and its rejection in magnetoelectric sensor design J. Appl. Phys. 106024512

[69] Salzer S, Durdaut P, Röbisch V, Meyners D, Quandt E, Höft M and Knöchel R 2017 Generalized Magnetic Frequency Conversion for Thin-Film Laminate Magnetoelectric Sensors IEEE Sens. J. 17 1373-83 
[70] Xu J, Zhuang X, Leung C, Staruch M., Finkel P. Li J and Viehland D 2018 Magnetoelectric gradiometer with enhanced vibration rejection efficiency under $\mathrm{H}$ field modulation J. Appl. Phys. 123104501

[71] Zhang J, Li P, Wen Y, He W, Yang J, Yang A, Lu C and Li W 2014 Enhanced sensitivity in magnetoelectric current-sensing devices with frequency up-conversion mechanism by modulating the magnetostrictive strain J. Appl. Phys. 115 17E505

[72] Shen Y, Gao J, Wang Y, Li J and Viehland D 2014 High non-linear magnetoelectric coefficient in Metglas/PMN-PT laminate composites under zero direct current magnetic bias J. Appl. Phys. 115094102

[73] Shen Y, Gao J, Wang Y, Finkel P, Li J and Viehland D 2013 Piezomagnetic straindependent non-linear magnetoelectric response enhancement by flux concentration effect Appl. Phys. Lett. 102172904

[74] Wang Y, Shen Y, Gao J, Li M, Li J and Viehland D 2013 Nonlinear magnetoelectric response of a Metglas/piezofiber laminate to a high-frequency bipolar AC magnetic field Appl. Phys. Lett. 102102905

[75] Wang Y, Finkel P, Li J and Viehland D 2014 Piezoelectric single crystal and magnetostrictive Metglas composites: Linear and nonlinear magnetoelectric coupling Appl. Phys. Lett. 104142909

[76] Li M, Wang Y, Shen Y, Gao J, Li J and Viehland D 2013 Structural dependence of nonlinear magnetoelectric effect for magnetic field detection by frequency modulation J. Appl. Phys. 114144501

[77] Huang J, O'Handley R C and Bono D 2003 New high-sensitivity hybrid magnetostrictive/electroactive magnetic field sensors Smart Structures and Materials 2003: Smart Sensor Technology and Measurement Systems vol 5050 (International Society for Optics and Photonics) pp 229-38

[78] Dai X, Wen Y, Li P, Yang J and Zhang G 2009 Modeling, characterization and fabrication of vibration energy harvester using Terfenol-D/PZT/Terfenol-D composite transducer Sens. Actuators Phys. $156350-8$

[79] Dai X, Wen Y, Li P, Yang J and Li M 2011 Energy harvesting from mechanical vibrations using multiple magnetostrictive/piezoelectric composite transducers Sens. Actuators Phys. 166 94-101

[80] Yang J, Wen Y, Li P and Bai X 2011 A magnetoelectric-based broadband vibration energy harvester for powering wireless sensors Sci. China Technol. Sci. 54 1419-27

[81] Bai X, Wen Y, Li P, Yang J, Peng X and Yue X 2014 Multi-modal vibration energy harvesting utilizing spiral cantilever with magnetic coupling Sens. Actuators Phys. 209 $78-86$

[82] Lin Z, Yang J, Zhao J, Zhao N, Liu J, Wen Y and Li P 2016 Enhanced Broadband Vibration Energy Harvesting Using a Multimodal Nonlinear Magnetoelectric Converter J. Electron. Mater. 45 3554-61 
[83] Yang J, Wen Y, Li P, Yue X and Bai X 2012 A bi-axial and wideband vibration energy harvester using magnetoelectric transducer 2012 IEEE International Ultrasonics Symposium 2012 IEEE International Ultrasonics Symposium pp 2404-7

[84] Moss S D, McLeod J E, Powlesland I G and Galea S C 2012 A bi-axial magnetoelectric vibration energy harvester Sens. Actuators Phys. 175 165-8

[85] Lin Z, Chen J, Li X, Li J, Liu J, Awais Q and Yang J 2016 Broadband and threedimensional vibration energy harvesting by a non-linear magnetoelectric generator Appl. Phys. Lett. 109253903

[86] Zhu Y and Zu J W 2012 A Magnetoelectric Generator for Energy Harvesting From the Vibration of Magnetic Levitation IEEE Trans. Magn. 48 3344-7

[87] Li M, Wen Y, Li P and Yang J 2013 A resonant frequency self-tunable rotation energy harvester based on magnetoelectric transducer Sens. Actuators Phys. 194 16-24

[88] Dai X 2016 An vibration energy harvester with broadband and frequency-doubling characteristics based on rotary pendulums Sens. Actuators Phys. 241 161-8

[89] Bayrashev A, Robbins W P and Ziaie B 2004 Low frequency wireless powering of microsystems using piezoelectric-magnetostrictive laminate composites Sens. Actuators Phys. $114244-9$

[90] Wang Y, Zhao X, Jiao J, Liu L, Di W, Luo H and Or S W 2010 Electrical resistance load effect on magnetoelectric coupling of magnetostrictive/piezoelectric laminated composite J. Alloys Compd. $\mathbf{5 0 0} 224-6$

[91] Gao J, Hasanyan D, Shen Y, Wang Y, Li J and Viehland D 2012 Giant resonant magnetoelectric effect in bi-layered Metglas/ $\mathrm{Pb}(\mathrm{Zr}, \mathrm{Ti}) \mathrm{O} 3$ composites J. Appl. Phys. 112104101

[92] Qiu J, Wen Y, Li P and Yang J 2012 Design and testing of piezoelectric energy harvester for powering wireless sensors of electric line monitoring system J. Appl. Phys. 111 07E510

[93] He W, Li P, Wen Y, Zhang J, Yang A and Lu C 2014 A Noncontact Magnetoelectric Generator for Energy Harvesting From Power Lines IEEE Trans. Magn. 50 1-4

[94] Leland E S, White R M and Wright P K 2006 Energy scavenging power sources for household electrical monitoring Proc. PowerMEMS vol 77

[95] He W, Li P, Wen Y, Zhang J, Yang A and Lu C 2014 Energy Harvesting From TwoWire Power Cords Using Magnetoelectric Transduction IEEE Trans. Magn. 50 1-5

[96] Dong S, Zhai J, Li J F, Viehland D and Priya S 2008 Multimodal system for harvesting magnetic and mechanical energy Appl. Phys. Lett. 93103511

[97] Lasheras A, Gutiérrez J, Reis S, Sousa D, Silva M, Martins P, Lanceros-Mendez S, Barandiarán J M, Shishkin D A and Potapov A P 2015 Energy harvesting device based on a metallic glass/PVDF magnetoelectric laminated composite Smart Mater. Struct. 24 065024 
[98] Zhou Y, Apo D J and Priya S 2013 Dual-phase self-biased magnetoelectric energy harvester Appl. Phys. Lett. 103192909

[99] Qiu J, Wen Y, Li P and Chen H 2015 Design and Optimization of a Tunable Magnetoelectric and Electromagnetic Hybrid Vibration-Based Generator for Wireless Sensor Networks IEEE Trans. Magn. 51 1-4

[100] Dong S, Li J F and Viehland D 2004 Voltage gain effect in a ring-type magnetoelectric laminate Appl. Phys. Lett. 84 4188-90

[101] Dong S, Li J F, Viehland D, Cheng J and Cross L E 2004 A strong magnetoelectric voltage gain effect in magnetostrictive-piezoelectric composite Appl. Phys. Lett. 85 $3534-6$

[102] Wang Y, Zhao X, Di W, Luo H and Or S W 2009 Magnetoelectric voltage gain effect in a long-type magnetostrictive/piezoelectric heterostructure Appl. Phys. Lett. 95 143503

[103] Lü L, Guo Y, Zhou J, Wang P, Liu P and Chen X 2011 Adjusting the voltage step-up ratio of a magnetoelectric composite transformer Chin. Sci. Bull. 56 700-3

[104] Zhou Y, Yan Y and Priya S 2014 Co-fired magnetoelectric transformer Appl. Phys. Lett. 104232906

[105] Zhai J, Gao J, Vreugd C D, Li J, Viehland D, Filippov A V, Bichurin M I, Drozdov D V, Semenov G A and Dong S X 2009 Magnetoelectric gyrator Eur. Phys. J. B 71383

[106] Zhuang X, Leung C M, Li J and Viehland D 2017 Evaluation of magnetomechanical conversion efficiencies in magnetoelectric gyrators AIP Adv. 8056607

[107] Leung C M, Zhuang X, Xu J, Srinivasan G, Li J and Viehland D 2016 Power conversion efficiency and resistance tunability in coil-magnetoelectric gyrators Appl. Phys. Lett. 109202907

[108] Dong S, Li J-F and Viehland D 2006 Magnetoelectric coupling, efficiency, and voltage gain effect in piezoelectric-piezomagnetic laminate composites Frontiers of Ferroelectricity (Springer, Boston, MA) pp 97-106

[109] Zhuang X VT Internal report No 5

[110] Leung C M, Zhuang X, Xu J, Li J, Srinivasan G and Viehland D 2017 Importance of composite parameters in enhanced power conversion efficiency of Terfenol-D/PZT magnetoelectric gyrators Appl. Phys. Lett. 110112904

[111] Zhuang X, Leung C M, Li J and Viehland D 2017 Power conversion process in magnetoelectric gyrators Appl. Phys. Lett. 111103902

[112] Leung C M, Zhuang X, Gao M, Tang X, Xu J, Li J, Zhang J, Srinivasan G and Viehland D 2017 Enhanced stability of magnetoelectric gyrators under high power conditions Appl. Phys. Lett. 111182901 
[113] Zhuang X, Leung C M, Sreenivasulu G, Gao M, Zhang J, Srinivasan G, Li J and Viehland D 2017 Upper limit for power conversion in magnetoelectric gyrators Appl. Phys. Lett. 111163902

[114] NASA, Mars inSight Mission. www.nasa.gov

[115] Department of Energy, What the smart grid means to America's future. www.smartgrid.gov

[116] T. Ohira, 2017 A battery-less electric roadway vehicle runs for the first time in the world Proc. IEEE Int. Conf. Microwaves Intelligent Mobility, Nagoya

[117] Departement of Defense, A MEchanically Based Antenna, https://www.darpa.mil 